\title{
Hardy spaces associated with different homogeneities and boundedness of composition operators
}

\author{
Yongsheng Han, Chincheng Lin, Guozhen Lu, \\ Zhuoping Ruan*and Eric T. Sawyer
}

\begin{abstract}
It is well known that standard Calderón-Zygmund singular integral operators with isotropic and nonisotropic homogeneities are bounded on the classical $H^{p}\left(\mathbb{R}^{m}\right)$ and nonisotropic $H_{h}^{p}\left(\mathbb{R}^{m}\right)$, respectively. In this paper, we develop a new Hardy space theory and prove that the composition of two Calderón-Zygmund singular integral operators with different homogeneities is bounded on this new Hardy space. Such a Hardy space has a multiparameter structure associated with the underlying mixed homogeneities arising from the two singular integral operators under consideration. The Calderón-Zygmund decomposition and an interpolation theorem hold on these new Hardy spaces.
\end{abstract}

\section{Introduction and statement of results}

The purpose of this paper is to develop a new Hardy space theory and prove that the composition of two Calderón-Zygmund singular integrals associated with different homogeneities is bounded on these new Hardy spaces. Indeed, the composition of operators was considered by Calderón and Zygmund when introducing the first generation of Calderón-Zygmund convolution operators. Calderón and Zygmund discovered that to compose two convolution operators, $T_{1}$ and $T_{2}$, it is enough to employ the product of the corresponding multipliers $m_{1}(\xi)$ and $m_{2}(\xi)$. However, the symbol $m_{3}(\xi)=m_{1}(\xi) m_{2}(\xi)$ does not necessarily have zero integral on the unit sphere, so they considered the algebra of operators $c I+T$, where $c$ is a constant, $I$ is the identity operator, and $T$ is the operator introduced by them. In 1965, Calderón considered again the problem of the symbolic calculus of the second generation of Calderón-Zygmund singular integral operators, with kernels $L_{1}(x, y)$ and $L_{2}(x, y)$

Mathematics Subject Classification (2010): Primary 42B30; Secondary 42B20.

Keywords: Hardy spaces, Calderón-Zygmund operators, discrete Calderón's identity, almost orthogonality estimates, discrete Littlewood-Paley-Stein square functions.

${ }^{*}$ Corresponding author. 
having minimal regularity with respect to $x$, and corresponding to operators $T_{1}$ and $T_{2}$. This problem reduced to the study of the commutator, which was the first nonconvolution operator considered in harmonic analysis.

In the present paper, we consider the composition of two operators associated with different homogeneities. To be more precise, let $e(\xi)$ be a function on $\mathbb{R}^{m}$ homogeneous of degree 0 in the isotropic sense and smooth away from the origin. Similarly, suppose that $h(\xi)$ is a function on $\mathbb{R}^{m}$ homogeneous of degree 0 in the nonisotropic sense related to the heat equation, and also smooth away from the origin. Then it is well known that the Fourier multipliers $T_{1}$ defined by $\widehat{T_{1}(f)}(\xi)=e(\xi) \widehat{f}(\xi)$ and $T_{2}$ given by $\widehat{T_{2}(f)}(\xi)=h(\xi) \widehat{f}(\xi)$ are both bounded on $L^{p}$ for $1<p<\infty$, and satisfy various other regularity properties such as being of weak-type $(1,1)$. It was well known that $T_{1}$ and $T_{2}$ are bounded on the classical isotropic and nonisotropic Hardy spaces, respectively. Rivieré in [20] asked the question: is the composition $T_{1} \circ T_{2}$ still of weak-type $(1,1)$ ? Phong and Stein in [19] answered this question and gave a necessary and sufficient condition for $T_{1} \circ T_{2}$ to be of weak-type $(1,1)$. The operators Phong and Stein studied are in fact compositions with different kinds of homogeneities which arise naturally in the $\bar{\partial}$-Neumann problem. This motivates the work in the present paper.

In order to describe more precisely the questions studied and the results in this paper, we begin by considering all functions and operators to be defined on $\mathbb{R}^{m}$. We write $\mathbb{R}^{m}=\mathbb{R}^{m-1} \times \mathbb{R}$ with $x=\left(x^{\prime}, x_{m}\right)$ where $x^{\prime} \in \mathbb{R}^{m-1}$ and $x_{m} \in \mathbb{R}$. We consider two kinds of homogeneities

$$
\delta:\left(x^{\prime}, x_{m}\right) \rightarrow\left(\delta x^{\prime}, \delta x_{m}\right), \quad \delta>0,
$$

and

$$
\delta:\left(x^{\prime}, x_{m}\right) \rightarrow\left(\delta x^{\prime}, \delta^{2} x_{m}\right), \quad \delta>0 .
$$

The first are the classical isotropic dilations occurring in the classical CalderónZygmund singular integrals, while the second are nonisotropic and are related to heat equations (also Heisenberg groups).

For $x=\left(x^{\prime}, x_{m}\right) \in \mathbb{R}^{m-1} \times \mathbb{R}$ we write $|x|_{e}=\left(\left|x^{\prime}\right|^{2}+\left|x_{m}\right|^{2}\right)^{1 / 2}$ and $|x|_{h}=$ $\left(\left|x^{\prime}\right|^{2}+\left|x_{m}\right|\right)^{1 / 2}$. We also use the notations $j \wedge k=\min \{j, k\}$ and $j \vee k=\max \{j, k\}$. The singular integrals considered in this paper are defined by:

Definition 1.1. A locally integrable function $\mathcal{K}_{1}$ on $\mathbb{R}^{m} /\{0\}$ is said to be a Calderón-Zygmund kernel associated with the isotropic homogeneity if

$$
\begin{aligned}
\left|\frac{\partial^{\alpha}}{\partial x^{\alpha}} \mathcal{K}_{1}(x)\right| & \leq A|x|_{e}^{-m-|\alpha|} \quad \text { for all }|\alpha| \geq 0, \\
\int_{r_{1}<|x|_{e}<r_{2}} \mathcal{K}_{1}(x) d x & =0 \text { for all } 0<r_{1}<r_{2}<\infty .
\end{aligned}
$$

We say that an operator $T_{1}$ is a Calderón-Zygmund singular integral operator associated with the isotropic homogeneity if $T_{1}(f)(x)=p \cdot v \cdot\left(\mathcal{K}_{1} * f\right)(x)$, where $\mathcal{K}_{1}$ satisfies conditions (1.1) and (1.2). 
Definition 1.2. Suppose $\mathcal{K}_{2} \in L_{\text {loc }}^{1}\left(\mathbb{R}^{m} \backslash\{0\}\right) . \mathcal{K}_{2}$ is said to be a Calderón-Zygmund kernel associated with the nonisotropic homogeneity if

$$
\begin{gathered}
\left|\frac{\partial^{\alpha}}{\partial\left(x^{\prime}\right)^{\alpha}} \frac{\partial^{\beta}}{\partial\left(x_{m}\right)^{\beta}} \mathcal{K}_{2}\left(x^{\prime}, x_{m}\right)\right| \leq B|x|_{h}^{-m-1-|\alpha|-2 \beta} \quad \text { for all }|\alpha| \geq 0, \beta \geq 0, \\
\int_{r_{1}<|x|_{h}<r_{2}} \mathcal{K}_{2}(x) d x=0 \quad \text { for all } 0<r_{1}<r_{2}<\infty .
\end{gathered}
$$

We say that an operator $T_{2}$ is a Calderón-Zygmund singular integral operator associated with the nonisotropic homogeneity if $T_{2}(f)(x)=p \cdot v \cdot\left(\mathcal{K}_{2} * f\right)(x)$, where $\mathcal{K}_{2}$ satisfies the conditions (1.3) and (1.4).

It is well known that any Calderón-Zygmund singular integral operator associated with the isotropic homogeneity is bounded on $L^{p}\left(\mathbb{R}^{m}\right)$ for $1<p<\infty$ and is also bounded on the classical Hardy space $H^{p}\left(\mathbb{R}^{m}\right)$ with $0<p \leq 1$. Here the classical Hardy space $H^{p}\left(\mathbb{R}^{m}\right)$ is that introduced by Fefferman and Stein in [5]. This space is associated with the isotropic homogeneity. To see this, let $\psi^{(1)} \in \mathcal{S}\left(\mathbb{R}^{m}\right)$ with

$$
\begin{aligned}
& \operatorname{supp} \widehat{\psi^{(1)}} \subseteq\left\{\left(\xi^{\prime}, \xi_{m}\right) \in \mathbb{R}^{m-1} \times \mathbb{R}: \frac{1}{2} \leq|\xi|_{e} \leq 2\right\} \\
& \sum_{j \in \mathbb{Z}}\left|\widehat{\psi^{(1)}}\left(2^{-j} \xi^{\prime}, 2^{-j} \xi_{m}\right)\right|^{2}=1 \quad \text { for all }\left(\xi^{\prime}, \xi_{m}\right) \in \mathbb{R}^{m-1} \times \mathbb{R} /\{(0,0)\}
\end{aligned}
$$

The Littlewood-Paley-Stein square function of $f \in \mathcal{S}^{\prime}\left(\mathbb{R}^{m}\right)$ is then defined by

$$
g(f)(x)=\left\{\sum_{j \in \mathbb{Z}}\left|\psi_{j}^{(1)} * f(x)\right|^{2}\right\}^{1 / 2},
$$

where $\psi_{j}^{(1)}\left(x^{\prime}, x_{m}\right)=2^{j m} \psi^{(1)}\left(2^{j} x^{\prime}, 2^{j} x_{m}\right)$. Note that the isotropic homogeneity is involved in $g(f)$. The classical Hardy space $H^{p}\left(\mathbb{R}^{m}\right)$ can then be characterized by

$$
H^{p}\left(\mathbb{R}^{m}\right)=\left\{f \in \mathcal{S}^{\prime} / \mathcal{P}\left(\mathbb{R}^{m}\right): g(f) \in L^{p}\left(\mathbb{R}^{m}\right)\right\},
$$

where $\mathcal{S}^{\prime} / \mathcal{P}$ denotes the space of distributions modulo polynomials. If $f \in H^{p}\left(\mathbb{R}^{m}\right)$, the $H^{p}$ norm of $f$ is defined by $\|f\|_{H^{p}}=\|g(f)\|_{L^{p}}$.

As we mentioned above, a Calderón-Zygmund singular integral operator associated with the nonisotropic homogeneity is bounded on $L^{p}, 1<p<\infty$. It is not bounded on the classical Hardy space but is bounded on the nonisotropic Hardy space. The nonisotropic Hardy space can also be characterized by the nonisotropic Littlewood-Paley-Stein square function. To be more precise, let $\psi^{(2)} \in \mathcal{S}\left(\mathbb{R}^{m}\right)$ with

$$
\begin{aligned}
& \operatorname{supp} \widehat{\psi^{(2)}} \subseteq\left\{\left(\xi^{\prime}, \xi_{m}\right) \in \mathbb{R}^{m-1} \times \mathbb{R}: \frac{1}{2} \leq|\xi|_{h} \leq 2\right\} \\
& \sum_{k \in \mathbb{Z}}\left|\widehat{\psi^{(2)}}\left(2^{-k} \xi^{\prime}, 2^{-2 k} \xi_{m}\right)\right|^{2}=1 \quad \text { for all }\left(\xi^{\prime}, \xi_{m}\right) \in \mathbb{R}^{m-1} \times \mathbb{R} \backslash\{(0,0)\}
\end{aligned}
$$


We then define $g_{h}(f)$, the nonisotropic Littlewood-Paley-Stein square function of $f \in \mathcal{S}^{\prime}\left(\mathbb{R}^{m}\right)$, by

$$
g_{h}(f)(x)=\left\{\sum_{k \in \mathbb{Z}}\left|\psi_{k}^{(2)} * f(x)\right|^{2}\right\}^{1 / 2},
$$

where $\psi_{k}^{(2)}\left(x^{\prime}, x_{m}\right)=2^{k(m+1)} \psi^{(2)}\left(2^{k} x^{\prime}, 2^{2 k} x_{m}\right)$. Note again that the nonisotropic homogeneity is involved in $g_{h}(f)$. The nonisotropic Hardy space $H_{h}^{p}\left(\mathbb{R}^{m}\right)$ then can be characterized by

$$
H_{h}^{p}\left(\mathbb{R}^{m}\right)=\left\{f \in \mathcal{S}^{\prime} / \mathcal{P}\left(\mathbb{R}^{m}\right): g_{h}(f) \in L^{p}\left(\mathbb{R}^{m}\right)\right\},
$$

and if $f \in H_{h}^{p}\left(\mathbb{R}^{m}\right)$, the $H_{h}^{p}$ norm of $f$ is defined by $\|f\|_{H_{h}^{p}}=\left\|g_{h}(f)\right\|_{L^{p}}$.

If $T_{1}$ and $T_{2}$ are Calderón-Zygmund singular integrals with isotropic and nonisotropic homogeneities, respectively, the composition $T_{1} \circ T_{2}$ is always bounded on $L^{p}, 1<p<\infty$, but, in general, bounded neither on the classical Hardy space $H^{p}\left(\mathbb{R}^{m}\right)$ nor on the nonisotropic Hardy space $H_{h}^{p}\left(\mathbb{R}^{m}\right)$. Our goal in this paper is to develop a new Hardy space theory associated with different homogeneities such that the composition $T_{1} \circ T_{2}$ is bounded on this new Hardy space. A new idea for how to achieve this is to establish the Littlewood-Paley-Stein theory associated with different homogeneities. More precisely, suppose that $\psi^{(1)}$ and $\psi^{(2)}$ are functions satisfying conditions in (1.5)-(1.6) and (1.7)-(1.8), respectively. Let $\psi_{j, k}(x)=\psi_{j}^{(1)} * \psi_{k}^{(2)}(x)$. Define a new Littlewood-Paley-Stein square function by

$$
\mathcal{G}_{\text {com }}(f)(x)=\left\{\sum_{j, k \in \mathbb{Z}}\left|\psi_{j, k} * f(x)\right|^{2}\right\}^{1 / 2} .
$$

We remark that a significant feature is that the multiparameter structure is involved in the above Littlewood-Paley-Stein square function. As in the classical case, it is not difficult to check that for $1<p<\infty$,

$$
\left\|\mathcal{G}_{\mathrm{com}}(f)\right\|_{L^{p}} \approx\|f\|_{L^{p}}
$$

The estimates above suggest us to define the $H^{p}$ norm of $f$ in terms of the $L^{p}$ norm of $\mathcal{G}_{\text {com }}(f)$ when $0<p \leq 1$. However, this continuous version of the Littlewood-Paley-Stein square function $\mathcal{G}_{\text {com }}(f)$ is convenient to deal with the case for $1<p<\infty$ but not for the case when $0<p \leq 1$. See further remark about this below. The crucial idea is to replace the continuous version $\mathcal{G}_{\text {com }}(f)$ by the discrete version $\mathcal{G}_{\psi}^{d}(f)$ as follows.

To define the discrete version $\mathcal{G}_{\psi}^{d}(f)$, the key tool is the discrete Calderón identity. To be more precise, we first recall the classical continuous Calderón identity on $L^{2}\left(\mathbb{R}^{m}\right)$. Let $\psi^{(1)}$ be a function satisfying the conditions (1.5) and (1.6). By taking the Fourier transform, we have the classical continuous Calderón identity

$$
f(x)=\sum_{j \in \mathbb{Z}} \psi_{j}^{(1)} * \psi_{j}^{(1)} * f(x)
$$


where the series converges in $L^{2}\left(\mathbb{R}^{m}\right)$ and in

$$
\mathcal{S}_{0}\left(\mathbb{R}^{m}\right):=\left\{f \in \mathcal{S}\left(\mathbb{R}^{m}\right): \int_{\mathbb{R}^{m}} f(x) x^{\alpha} d x=0 \text { for any }|\alpha| \geq 0\right\} .
$$

Note that the Fourier transforms of both $\psi_{j}^{(1)}$ and $\psi_{j}^{(1)} * f$ are compactly supported. Using a similar idea as in the Shannon sampling theorem, one can decompose $\psi_{j}^{(1)} * \psi_{j}^{(1)} * f(x)$ as

$$
\sum_{\ell \in \mathbb{Z}^{m}} \psi_{j}^{(1)}\left(x-2^{-j} \ell\right)\left(\psi_{j}^{(1)} * f\right)\left(2^{-j} \ell\right)
$$

Then the classical discrete Calderón identity is given by

$$
f(x)=\sum_{j \in \mathbb{Z}} \sum_{\ell \in \mathbb{Z}^{m}} \psi_{j}^{(1)}\left(x-2^{-j} \ell\right)\left(\psi_{j}^{(1)} * f\right)\left(2^{-j} \ell\right),
$$

where the series converges in $L^{2}\left(\mathbb{R}^{m}\right), \mathcal{S}_{0}\left(\mathbb{R}^{m}\right)$ and $\mathcal{S}_{0}^{\prime}\left(\mathbb{R}^{m}\right)$. See [10] and [11] for more details.

Now by considering $\psi_{j, k}=\psi_{j}^{(1)} * \psi_{k}^{(2)}$ and taking the Fourier transform, we obtain the continuous Calderón identity

$$
f(x)=\sum_{j, k \in \mathbb{Z}} \psi_{j, k} * \psi_{j, k} * f(x),
$$

where the series converges in $L^{2}\left(\mathbb{R}^{m}\right), \mathcal{S}_{0}\left(\mathbb{R}^{m}\right)$ and $\mathcal{S}_{0}^{\prime}\left(\mathbb{R}^{m}\right)$. Furthermore, we will prove the following discrete Calderón identity.

Theorem 1.3. Suppose that $\psi^{(1)}$ and $\psi^{(2)}$ are functions satisfying conditions in (1.5)-(1.6) and (1.7)-(1.8), respectively. Let $\psi_{j, k}(x)=\psi_{j}^{(1)} * \psi_{k}^{(2)}(x)$. Then

$f\left(x^{\prime}, x_{m}\right)=\sum_{j, k \in \mathbb{Z}} \sum_{\left(\ell^{\prime}, \ell_{m}\right) \in \mathbb{Z}^{m-1} \times \mathbb{Z}} 2^{-(m-1)(j \wedge k)} 2^{-(j \wedge 2 k)}\left(\psi_{j, k} * f\right)\left(2^{-(j \wedge k)} \ell^{\prime}, 2^{-(j \wedge 2 k)} \ell_{m}\right)$

$$
\times \psi_{j, k}\left(x^{\prime}-2^{-(j \wedge k)} \ell^{\prime}, x_{m}-2^{-(j \wedge 2 k)} \ell_{m}\right),
$$

where the series converges in $L^{2}\left(\mathbb{R}^{m}\right), \mathcal{S}_{0}\left(\mathbb{R}^{m}\right)$ and $\mathcal{S}_{0}^{\prime}\left(\mathbb{R}^{m}\right)$.

This discrete Calderón identity leads to the following discrete LittlewoodPaley-Stein square function.

Definition 1.4. For $f \in \mathcal{S}_{0}^{\prime}\left(\mathbb{R}^{m}\right), \mathcal{G}_{\psi}^{d}(f)$, the discrete Littlewood-Paley-Stein square function of $f$, is defined by

$$
\begin{gathered}
\mathcal{G}_{\psi}^{d}(f)\left(x^{\prime}, x_{m}\right)=\left\{\sum_{j, k \in \mathbb{Z}} \sum_{\left(\ell^{\prime}, \ell_{m}\right) \in \mathbb{Z}^{m-1} \times \mathbb{Z}}\left|\left(\psi_{j, k} * f\right)\left(2^{-(j \wedge k)} \ell^{\prime}, 2^{-(j \wedge 2 k)} \ell_{m}\right)\right|^{2}\right. \\
\left.\times \chi_{I}\left(x^{\prime}\right) \chi_{J}\left(x_{m}\right)\right\}^{1 / 2}
\end{gathered}
$$

where the $I$ are dyadic cubes in $\mathbb{R}^{m-1}$, and the $J$ are dyadic intervals in $\mathbb{R}$ with side lengths $\ell(I)=2^{-(j \wedge k)}$ and $\ell(J)=2^{-(j \wedge 2 k)}$, and the lower left corners of $I$ and the left endpoints of $J$ are $2^{-(j \wedge k)} \ell^{\prime}$ and $2^{-(j \wedge 2 k)} \ell_{m}$, respectively. 
Now we formally define the Hardy spaces associated with two different homogeneities:

Definition 1.5. Let $0<p \leq 1$. $H_{\text {com }}^{p}\left(\mathbb{R}^{m}\right)=\left\{f \in \mathcal{S}_{0}^{\prime}\left(\mathbb{R}^{m}\right): \mathcal{G}_{\psi}^{d}(f) \in L^{p}\left(\mathbb{R}^{m}\right)\right\}$. If $f \in H_{\mathrm{com}}^{p}\left(\mathbb{R}^{m}\right)$, the norm of $f$ is defined by $\|f\|_{H_{\mathrm{com}}^{p}\left(\mathbb{R}^{m}\right)}=\left\|\mathcal{G}_{\psi}^{d}(f)\right\|_{L^{p}\left(\mathbb{R}^{m}\right)}$.

Note that, as mentioned above for the Littlewood-Paley-Stein square function, the multiparameter structures are involved again in the discrete Calderón identity and the Hardy spaces $H_{\text {com }}^{p}\left(\mathbb{R}^{m}\right)$. To see that these Hardy spaces are well defined, we need to show that $H_{\text {com }}^{p}\left(\mathbb{R}^{m}\right)$ is independent of the choice of the functions $\psi^{(1)}$ and $\psi^{(2)}$. This will follow directly from the following theorem:

Theorem 1.6. If $\varphi$ satisfies the same conditions as $\psi$, then for $0<p \leq 1$ and $f \in \mathcal{S}_{0}^{\prime}\left(\mathbb{R}^{m}\right)$,

$$
\left\|\mathcal{G}_{\psi}^{d}(f)\right\|_{L^{p}\left(\mathbb{R}^{m}\right)} \approx\left\|\mathcal{G}_{\varphi}^{d}(f)\right\|_{L^{p}\left(\mathbb{R}^{m}\right)} .
$$

We would like to point out that one can define the Hardy space $H_{\text {com }}^{p}\left(\mathbb{R}^{m}\right)$ in terms of $\mathcal{G}_{\text {com }}(f)$, the Littlewood-Paley-Stein square function. Then one has to show Theorem 1.6, that is, the norm of $H_{\text {com }}^{p}\left(\mathbb{R}^{m}\right)$ defined by $\mathcal{G}_{\text {com }}(f)$ must be independent of the choice of the function $\psi$ in $\mathcal{G}_{\text {com }}(f)$. However, such a proof of Theorem 1.6 with $\mathcal{G}^{d}(f)$ replaced by $\mathcal{G}_{\text {com }}(f)$ is more complicated. This is why, instead of using $\mathcal{G}_{\text {com }}(f)$, we decide to use $\mathcal{G}_{\psi}^{d}(f)$ to define the Hardy space $H_{\text {com }}^{p}\left(\mathbb{R}^{m}\right)$. Indeed, by applying an argument similar to that in [10], one can also show that for all $0<p<\infty$,

$$
\left\|\mathcal{G}_{\mathrm{com}}(f)\right\|_{p} \approx\left\|\mathcal{G}_{\psi}^{d}(f)\right\|_{p} .
$$

We omit the proof and refer the reader to [10] for the details.

We now state the main results of this paper.

Theorem 1.7. Let $T_{1}$ and $T_{2}$ be Calderón-Zygmund singular integral operators with the isotropic and nonisotropic homogeneity, respectively. Then for $0<p \leq 1$, the composition operator $T=T_{1} \circ T_{2}$ is bounded on $H_{\mathrm{com}}^{p}\left(\mathbb{R}^{m}\right)$.

It is well known that the atomic decomposition of the classical Hardy spaces is the main tool for studying the $H^{p}-L^{p}$ boundedness of classical Calderón-Zygmund operators. See [4], [6], [9] and [12]. However, to get an atomic decomposition for the Hardy space $H_{\mathrm{com}}^{p}\left(\mathbb{R}^{m}\right)$ with multiparameter structures, as in the classical case, one needs first to establish Journé's covering lemma in this setting. See [1], $[2],[3],[17],[7],[8]$, and [18] for more details. Our approach is quite different from this scheme. Indeed, we will prove the following theorem:

Theorem 1.8. Let $0<p \leq 1$. If $f \in L^{2}\left(\mathbb{R}^{m}\right) \cap H_{\mathrm{com}}^{p}\left(\mathbb{R}^{m}\right)$, then there is a constant $C=C(p)$ such that

$$
\|f\|_{L^{p}\left(\mathbb{R}^{m}\right)} \leq C\|f\|_{H_{\mathrm{com}}^{p}\left(\mathbb{R}^{m}\right)},
$$

where the constant $C$ is independent of $f$.

We remark that the proof of the above theorem does not use an atomic decomposition and hence Journé's covering lemma is not required. 
As a consequence, we obtain:

Theorem 1.9. Let $0<p \leq 1$. Suppose that $T$ is a composition of $T_{1}$ and $T_{2}$ as given in Theorem 1.7. Then $T$ extends to a bounded operator from $H_{\mathrm{com}}^{p}\left(\mathbb{R}^{m}\right)$ to $L^{p}\left(\mathbb{R}^{m}\right)$.

Next we provide the Calderón-Zygmund decomposition and prove an interpolation theorem on $H_{\text {com }}^{p}\left(\mathbb{R}^{m}\right)$. We note that $H_{\text {com }}^{p}\left(\mathbb{R}^{m}\right)=L^{p}\left(\mathbb{R}^{m}\right)$ for $1<p<\infty$.

Theorem 1.10 (Calderón-Zygmund decomposition for $H_{\mathrm{com}}^{p}$ ). Let $0<p_{2} \leq 1$, $p_{2}<p<p_{1}<\infty$, and let $\alpha>0$ and $f \in H_{\mathrm{com}}^{p}$ be given. Then we may write $f=g+b$, where $g \in H_{\mathrm{com}}^{p_{1}}$ and $b \in H_{\mathrm{com}}^{p_{2}}$ are such that $\|g\|_{H_{\mathrm{com}}^{p_{1}}}^{p_{1}} \leq C \alpha^{p_{1}-p}\|f\|_{H_{\mathrm{com}}^{p}}^{p}$ and $\|b\|_{H_{\mathrm{com}}^{p_{2}}}^{p_{2}} \leq C \alpha^{p_{2}-p}\|f\|_{H_{\mathrm{com}}^{p}}^{p}$, where $C$ is an absolute constant.

Theorem 1.11 (Interpolation theorem on $H_{\mathrm{com}}^{p}$ ). Let $0<p_{2}<p_{1}<\infty$ and let $T$ be a linear operator which is bounded from $H_{\mathrm{com}}^{p_{2}}$ to $L^{p_{2}}$ and bounded from $H_{\mathrm{com}}^{p_{1}}$ to $L^{p_{1}}$. Then $T$ is bounded from $H_{\mathrm{com}}^{p}$ to $L^{p}$ for all $p_{2}<p<p_{1}$. Similarly, if $T$ is bounded on $H_{\mathrm{com}}^{p_{2}}$ and $H_{\mathrm{com}}^{p_{1}}$, then $T$ is bounded on $H_{\mathrm{com}}^{p}$ for all $p_{2}<p<p_{1}$.

Before we end this section, several remarks are in order. First, as mentioned before, the continuous version of the Littlewood-Paley-Stein square function $\mathcal{G}_{\text {com }}(f)$ is convenient for dealing with the case $1<p<\infty$, but not for the case $0<p \leq 1$. However, we can still use this continuous version $\mathcal{G}_{\text {com }}(f)$ to define the Hardy spaces $H_{\text {com }}^{p}\left(\mathbb{R}^{m}\right)$ for $0<p \leq 1$. More precisely, suppose that $\psi^{(1)} \in \mathcal{S}_{0}$ satisfies

$$
\int_{0}^{\infty}\left|\widehat{\psi^{(1)}}\left(t \xi^{\prime}, t \xi_{m}\right)\right|^{2} \frac{d t}{t}=1 \quad \text { for all }\left(\xi^{\prime}, \xi_{m}\right) \in \mathbb{R}^{m-1} \times \mathbb{R} /\{(0,0)\}
$$

and $\psi^{(2)} \in \mathcal{S}_{0}$ satisfies

$$
\int_{0}^{\infty}\left|\widehat{\psi^{(2)}}\left(s \xi^{\prime}, s^{2} \xi_{m}\right)\right|^{2} \frac{d s}{s}=1 \quad \text { for all }\left(\xi^{\prime}, \xi_{m}\right) \in \mathbb{R}^{m-1} \times \mathbb{R} /\{(0,0)\}
$$

Set $\psi_{t, s}=\psi_{t}^{(1)} * \psi_{s}^{(2)}$, where $\psi_{t}^{(1)}\left(x^{\prime}, x_{m}\right)=t^{-m} \psi^{(1)}\left(x^{\prime} / t, x_{m} / t\right)$ and $\psi_{s}^{(2)}\left(x^{\prime}, x_{m}\right)=$ $s^{-m-1} \psi^{(2)}\left(x^{\prime} / s, x_{m} / s^{2}\right)$. Then, using the ideas in this paper, one can argue that the $H_{\text {com }}^{p}\left(\mathbb{R}^{m}\right)$ norm of the $f$ defined in Definition 1.5 is equivalent to

$$
\left\|\left\{\int_{0}^{\infty} \int_{0}^{\infty}\left|\psi_{t, s} * f\left(x^{\prime}, x_{m}\right)\right|^{2} \frac{d t}{t} \frac{d s}{s}\right\}^{1 / 2}\right\|_{L^{p}} .
$$

Second, in this paper we restrict our attention to the above two very specific dilations. However, all the results in this paper can be carried over to the composition with more singular integral operators associated with more general nonisotropic homogeneities. To see this, let $T_{i}(f)(x)=p \cdot v \cdot K_{i} * f(x), 1 \leq i \leq n$, be singular integral operators associated with nonisotropic dilations given by

$$
\delta_{i}:\left(x_{1}, x_{2}, \ldots, x_{m}\right) \rightarrow\left(\delta_{i}^{\lambda_{i, 1}} x_{1}, \delta_{i}^{\lambda_{i, 2}} x_{2}, \ldots, \delta_{i}^{\lambda_{i, m}} x_{m}\right)
$$

for $\delta_{i}>0, \lambda_{i, \ell}>0,1 \leq i \leq n$ and $1 \leq \ell \leq m$. 
For $x \in \mathbb{R}^{m}$ we write

$$
|x|_{i}=\left(\left|x_{1}\right|^{2 / \lambda_{i, 1}}+\left|x_{2}\right|^{2 / \lambda_{i, 2}}+\cdots+\left|x_{m}\right|^{2 / \lambda_{i, m}}\right)^{1 / 2} \text {. }
$$

Let $\psi^{(i)} \in \mathcal{S}\left(\mathbb{R}^{m}\right)$ with

$$
\operatorname{supp} \widehat{\psi^{(i)}} \subseteq\left\{\left(\xi_{1}, \xi_{2}, \ldots, \xi_{m}\right) \in \mathbb{R}^{m}: \frac{1}{2} \leq|\xi|_{i} \leq 2\right\}
$$

and

$$
\sum_{j_{i} \in \mathbb{Z}}\left|\widehat{\psi^{(i)}}\left(2^{-j_{i} \lambda_{i, 1}} \xi_{1}, 2^{-j_{i} \lambda_{i, 2}} \xi_{2}, \ldots, 2^{-j_{i} \lambda_{i, m}} \xi_{m}\right)\right|^{2}=1
$$

for all $\left(\xi_{1}, \xi_{2}, \ldots, \xi_{m}\right) \in \mathbb{R}^{m} /\{0\}$. Set $\psi_{j_{1}, j_{2}, \ldots, j_{n}}(x)=\psi_{j_{1}}^{(1)} * \psi_{j_{2}}^{(2)} * \cdots * \psi_{j_{n}}^{(n)}(x)$, where

$$
\psi_{j_{i}}^{(i)}(x)=2^{j_{i}\left(\lambda_{i, 1}+\lambda_{i, 2}+\cdots+\lambda_{i, m}\right)} \psi^{(i)}\left(2^{j_{i} \lambda_{i, 1}} x_{1}, 2^{j_{i} \lambda_{i, 2}} x_{2}, \ldots, 2^{j_{i} \lambda_{i, m}} x_{m}\right) .
$$

Define a Littlewood-Paley-Stein square function by

$$
\widetilde{\mathcal{G}}_{\mathrm{com}}(f)(x)=\left\{\sum_{j_{1}, j_{2}, \cdot \cdot, j_{n} \in \mathbb{Z}}\left|\psi_{j_{1}, j_{2}, \cdot \cdot, j_{n}} * f(x)\right|^{2}\right\}^{1 / 2} .
$$

Applying the same strategy as in this paper, one can develop the Hardy space theory associated with these more general nonisotropic dilations. The details of the proofs seem to be rather lengthy to be written out. Therefore, we shall not discuss these in more detail in this paper.

Third, the regularity conditions on kernels can be weakened if one considers the $H_{\text {com }}^{p}\left(\mathbb{R}^{m}\right)$ boundedness only for a certain range of $p$.

Finally, we would like to remark that the method of discrete Littlewood-PaleyStein analysis in the multiparameter settings employed in this paper has been used previously in a number of other cases. It first appeared in [13], where the theory of the multiparameter Hardy spaces associated with the flag singular integrals was developed, and in [14], where the discrete Littlewood-Paley-Stein theory was established in the multiparameter structure associated with the Zygmund dilation (see also the expository article [15]). Recently, the implicit multiparameter Hardy space and the Marcinkiewicz multiplier theory on the Heisenberg group have been developed in [16]. This method allows us to avoid using the deep Journé covering lemma to prove the boundedness of multiparameter singular integrals from the Hardy spaces.

Section 1 deals with Theorem 1.3. The proof of Theorem 1.6 is given in Section 3. The method of the proof will be applied to the proof of Theorem 1.8 and Theorem 1.10. To show Theorem 1.7, we provide a discrete Calderón-type identity, Theorem 4.1, which has its own interest. These will be given in Section 4. Theorem 1.8 and Theorem 1.9 are proved in Section 5. In the last section, we prove the Calderón-Zygmund decomposition and the interpolation theorems.

Acknowledgements. The authors wish to express their sincere thanks to the referee for his/her valuable comments and suggestions. 


\section{Proof of Theorem 1.3}

As mentioned in the previous section, by taking the Fourier transform, we obtain the continuous Calderón identity

$$
f(x)=\sum_{j, k \in \mathbb{Z}} \psi_{j, k} * \psi_{j, k} * f(x),
$$

where the convergence of series in $L^{2}\left(\mathbb{R}^{m}\right), \mathcal{S}_{0}\left(\mathbb{R}^{m}\right)$ and $\mathcal{S}_{0}^{\prime}\left(\mathbb{R}^{m}\right)$ follows from the results in the classical case. See [10] and [11] for more details.

To get a discrete version of Calderón's identity, we need to decompose $\psi_{j, k} *$ $\psi_{j, k} * f$ in (2.1). Using a method similar to that in [11], set $g=\psi_{j, k} * f$ and $h=\psi_{j, k}$. The Fourier transforms of $g$ and $h$ are given by

$$
\widehat{g}\left(\xi^{\prime}, \xi_{m}\right)=\widehat{\psi^{(1)}}\left(2^{-j} \xi^{\prime}, 2^{-j} \xi_{m}\right) \widehat{\psi^{(2)}}\left(2^{-k} \xi^{\prime}, 2^{-2 k} \xi_{m}\right) \widehat{f}\left(\xi^{\prime}, \xi_{m}\right)
$$

and

$$
\widehat{h}\left(\xi^{\prime}, \xi_{m}\right)=\widehat{\psi^{(1)}}\left(2^{-j} \xi^{\prime}, 2^{-j} \xi_{m}\right) \widehat{\psi^{(2)}}\left(2^{-k} \xi^{\prime}, 2^{-2 k} \xi_{m}\right) .
$$

Note that the Fourier transforms of $g$ and $h$ are both compactly supported. More precisely,

$$
\operatorname{supp} \widehat{g}, \operatorname{supp} \widehat{h} \subseteq\left\{\left(\xi^{\prime}, \xi_{m}\right) \in \mathbb{R}^{m-1} \times \mathbb{R}:\left|\xi^{\prime}\right| \leq 2^{j \wedge k} \pi,\left|\xi_{m}\right| \leq 2^{j \wedge 2 k} \pi\right\} .
$$

We first expand $\widehat{g}$ in a Fourier series on the rectangle $R_{j, k}=\left\{\xi^{\prime} \in \mathbb{R}^{m-1}, \xi_{m} \in \mathbb{R}\right.$ : $\left.\left|\xi^{\prime}\right| \leq 2^{j \wedge k} \pi,\left|\xi_{m}\right| \leq 2^{j \wedge 2 k} \pi\right\}$ :

$$
\begin{gathered}
\widehat{g}\left(\xi^{\prime}, \xi_{m}\right)=\sum_{\left(\ell^{\prime}, \ell_{m}\right) \in \mathbb{Z}^{m-1} \times \mathbb{Z}} 2^{-(m-1)(j \wedge k)} 2^{-(j \wedge 2 k)}(2 \pi)^{-m} \\
\times \int_{R_{j, k}} \widehat{g}\left(\eta^{\prime}, \eta_{m}\right) e^{i\left(2^{-(j \wedge k)} \ell^{\prime} \cdot \eta^{\prime}+2^{-(j \wedge 2 k)} \ell_{m} \eta_{m}\right)} d \eta^{\prime} d \eta_{m} \\
\times e^{-i\left(2^{-(j \wedge k)} \ell^{\prime} \cdot \xi^{\prime}+2^{-(j \wedge 2 k)} \ell_{m} \xi_{m}\right)},
\end{gathered}
$$

and then replace $R_{j, k}$ by $\mathbb{R}^{m}$ since $\widehat{g}$ is supported in $R_{j, k}$. Finally, we obtain

$$
\begin{array}{r}
\widehat{g}\left(\xi^{\prime}, \xi_{m}\right)=\sum_{\left(\ell^{\prime}, \ell_{m}\right) \in \mathbb{Z}^{m-1} \times \mathbb{Z}} 2^{-(m-1)(j \wedge k)} 2^{-(j \wedge 2 k)} g\left(2^{-(j \wedge k)} \ell^{\prime}, 2^{-(j \wedge 2 k)} \ell_{m}\right) \\
\times e^{-i\left(2^{-(j \wedge k)} \ell^{\prime} \cdot \xi^{\prime}+2^{-(j \wedge 2 k)} \ell_{m} \xi_{m}\right)} .
\end{array}
$$

Multiplying both sides by $\widehat{h}\left(\xi^{\prime}, \xi_{m}\right)$ yields

$$
\begin{gathered}
\widehat{g}\left(\xi^{\prime}, \xi_{m}\right) \widehat{h}\left(\xi^{\prime}, \xi_{m}\right)=\sum_{\left(\ell^{\prime}, \ell_{m}\right) \in \mathbb{Z}^{m-1} \times \mathbb{Z}} 2^{-(m-1)(j \wedge k)} 2^{-(j \wedge 2 k)} g\left(2^{-(j \wedge k)} \ell^{\prime}, 2^{-(j \wedge 2 k)} \ell_{m}\right) \\
\times \widehat{h}\left(\xi^{\prime}, \xi_{m}\right) e^{-i\left(2^{-(j \wedge k)} \ell^{\prime} \cdot \xi^{\prime}+2^{-(j \wedge 2 k)} \ell_{m} \xi_{m}\right)} .
\end{gathered}
$$

Note that

$$
\widehat{h}\left(\xi^{\prime}, \xi_{m}\right) e^{-i\left(2^{-(j \wedge k)} \ell^{\prime} \cdot \xi^{\prime}+2^{-(j \wedge 2 k)} \ell_{m} \xi_{m}\right)}=\widehat{h}\left(\cdot-2^{-(j \wedge k)} \ell^{\prime}, \cdot-2^{-(j \wedge 2 k)} \ell_{m}\right)\left(\xi^{\prime}, \xi_{m}\right) .
$$


Therefore, applying the identity $g * h=(\widehat{g} \widehat{h})^{\vee}$ implies that

$$
\begin{gathered}
(g * h)\left(x^{\prime}, x_{m}\right)=\sum_{\left(\ell^{\prime}, \ell_{m}\right) \in \mathbb{Z}^{m-1} \times \mathbb{Z}} 2^{-(m-1)(j \wedge k)} 2^{-(j \wedge 2 k)} g\left(2^{-(j \wedge k)} \ell^{\prime}, 2^{-(j \wedge 2 k)} \ell_{m}\right) \\
\times h\left(x^{\prime}-2^{-(j \wedge k)} \ell^{\prime}, x_{m}-2^{-(j \wedge 2 k)} \ell_{m}\right) .
\end{gathered}
$$

Substituting $g$ by $\psi_{j, k} * f$ and $h$ by $\psi_{j, k}$ in Calderón's identity (2.1) gives the discrete Calderón identity (1.12), and the convergence of the series in $L^{2}\left(\mathbb{R}^{m}\right)$.

It remains to prove that the series in $(1.12)$ converges in $\mathcal{S}_{0}\left(\mathbb{R}^{m}\right)$. To do this, it suffices to show that

$$
\begin{gathered}
\sum_{\substack{|j| \geq N_{1} \text { or }|k| \geq N_{2} \\
(2.3)}} \sum_{\left(\ell^{\prime}, \ell_{m}\right) \in \mathbb{Z}^{m-1} \times \mathbb{Z}} 2^{-(m-1)(j \wedge k)} 2^{-(j \wedge 2 k)}\left(\psi_{j, k} * f\right)\left(2^{-(j \wedge k)} \ell^{\prime}, 2^{-(j \wedge 2 k)} \ell_{m}\right) \\
\times \psi_{j, k}\left(x^{\prime}-2^{-(j \wedge k)} \ell^{\prime}, x_{m}-2^{-(j \wedge 2 k)} \ell_{m}\right)
\end{gathered}
$$

tend to zero in $\mathcal{S}_{0}\left(\mathbb{R}^{m}\right)$ as $N_{1}$ and $N_{2}$ tend to infinity.

For the sake of convenience, we write $x_{I}=2^{-(j \wedge k)} \ell^{\prime}$ and $x_{J}=2^{-(j \wedge 2 k)} \ell_{m}$. Let $I$ be dyadic cubes in $\mathbb{R}^{m-1}$ and let $J$ be dyadic intervals in $\mathbb{R}$ with side lengths $\ell(I)=2^{-(j \wedge k)}$ and $\ell(J)=2^{-(j \wedge 2 k)}$, and let the lower left corners of $I$ and the left endpoints of $J$ be $x_{I}$ and $x_{J}$, respectively. Then the above limit will follow from the following estimates: for any fixed $j, k$ and any given integer $M>0,|\alpha| \geq 0$, there exists a constant $C=C(M, \alpha)>0$ which is independent of $j$ and $k$ such that

$$
\begin{aligned}
\left|\sum_{I \times J}\right| I|| J \mid\left(\psi_{j, k} * f\right)\left(x_{I}, x_{J}\right)\left(D^{\alpha} \psi_{j, k}\right) & \left(x^{\prime}-x_{I}, x_{m}-x_{J}\right) \mid \\
& \leq C 2^{-|j|} 2^{-|k|}\left(1+\left|x^{\prime}\right|+\left|x_{m}\right|\right)^{-M}
\end{aligned}
$$

To show (2.4), we apply the classical almost orthogonality argument. To be more precise, for any given positive integers $L_{1}$ and $L_{2}$, there exists a constant $C=$ $C\left(L_{1}, L_{2}\right)>0$ such that

$$
\left|\psi_{j}^{(1)} * \psi_{j^{\prime}}^{(1)}\left(x^{\prime}, x_{m}\right)\right| \leq C \frac{2^{-\left|j-j^{\prime}\right| L_{1}} 2^{\left(j \wedge j^{\prime}\right) m}}{\left(1+2^{\left(j \wedge j^{\prime}\right)}\left|x^{\prime}\right|+2^{\left(j \wedge j^{\prime}\right)}\left|x_{m}\right|\right)^{L_{2}}}
$$

and

$$
\left|\psi_{k}^{(2)} * \psi_{k^{\prime}}^{(2)}\left(x^{\prime}, x_{m}\right)\right| \leq C \frac{2^{-\left|k-k^{\prime}\right| L_{1}} 2^{\left(k \wedge k^{\prime}\right)(m+1)}}{\left(1+2^{\left(k \wedge k^{\prime}\right)}\left|x^{\prime}\right|+2^{2\left(k \wedge k^{\prime}\right)}\left|x_{m}\right|\right)^{L_{2}}} .
$$

Applying (2.6) with $\psi_{0}^{(2)}=f, L_{1}=L+2 M+m+1$ and $L_{2}=M$, where $L$ and $M$ are any fixed positive integers, we obtain

$$
\begin{aligned}
\left|\left(\psi_{k}^{(2)} * f\right)\left(x^{\prime}, x_{m}\right)\right| & \leq C 2^{-|k|(L+2 M+m+1)} \frac{2^{-(k \wedge 0)(m+1)}}{\left(1+2^{(k \wedge 0)}\left|x^{\prime}\right|+2^{(2 k \wedge 0)}\left|x_{m}\right|\right)^{M}} \\
& \leq C 2^{-|k| L} \frac{1}{\left(1+\left|x^{\prime}\right|+\left|x_{m}\right|\right)^{M}},
\end{aligned}
$$


where the last inequality is obvious if $k \geq 0$, and when $k \leq 0$,

$$
\frac{2^{-k(m+1)}}{\left(1+2^{k}\left|x^{\prime}\right|+2^{2 k}\left|x_{m}\right|\right)^{M}} \leq 2^{|k|(2 M+m+1)} \frac{1}{\left(1+\left|x^{\prime}\right|+\left|x_{m}\right|\right)^{M}} .
$$

Note that $\psi_{k}^{(2)} * f \in \mathcal{S}_{0}\left(\mathbb{R}^{m}\right)$. Similarly, we have that

$$
\left|\left(\psi_{j}^{(1)} *\left(\psi_{k}^{(2)} * f\right)\right)\left(u^{\prime}, u_{m}\right)\right| \leq C 2^{-|k| L} 2^{-|j| L} \frac{1}{\left(1+\left|u^{\prime}\right|+\left|u_{m}\right|\right)^{M}} .
$$

From the size conditions of the functions $\psi^{(1)}$ and $\psi^{(2)}$, we have that for any fixed large $M$,

$$
\begin{aligned}
&\left|D^{\alpha} \psi_{j, k}\left(u^{\prime}, u_{m}\right)\right|=\left|D^{\alpha}\left(\psi_{j}^{(1)} * \psi_{k}^{(2)}\right)\left(u^{\prime}, u_{m}\right)\right| \\
& \leq \\
& \quad \times \frac{2^{|j||\alpha|+2|k||\alpha|} \int \frac{2^{j(m-1)}}{\left(1+2^{j}\left|u^{\prime}-v^{\prime}\right|+2^{j}\left|u_{m}-v_{m}\right|\right)^{M}}}{\left(1+2^{k}\left|v^{\prime}\right|+2^{2 k}\left|v_{m}\right|\right)^{M}} d v^{\prime} d v_{m} \\
& \leq C 2^{|j||\alpha|+2|k||\alpha|} \frac{2^{(j \wedge k)(m-1)} 2^{(j \wedge 2 k)}}{\left(1+2^{j \wedge k}\left|u^{\prime}\right|+2^{j \wedge 2 k}\left|u_{m}\right|\right)^{M}} \\
& \leq C 2^{|j|(M+m+|\alpha|)} 2^{|k|(2 M+2+2|\alpha|)} \frac{1}{\left(1+\left|u^{\prime}\right|+\left|u_{m}\right|\right)^{M}} .
\end{aligned}
$$

The estimates in (2.7) and (2.8) yield

$$
\begin{aligned}
\left|\sum_{I \times J}\right| I|| J \mid( & \left.D^{\alpha} \psi_{j, k}\right)\left(x^{\prime}-x_{I}, x_{m}-x_{J}\right)\left(\psi_{j, k} * f\right)\left(x_{I}, x_{J}\right) \mid \\
\leq & C 2^{-|k|(L-2 M-2|\alpha|-2)} 2^{-|j|(L-M-m-|\alpha|)} \\
\quad & \times \sum_{I \times J}|I||J| \frac{1}{\left(1+\left|x_{I}\right|+\left|x_{J}\right|\right)^{M}\left(1+\left|x^{\prime}-x_{I}\right|+\left|x_{m}-x_{J}\right|\right)^{M}} \\
= & C 2^{-|k|(L-2 M-2|\alpha|-2)} 2^{-|j|(L-M-m-|\alpha|)} \\
& \times \sum_{I \times J} \int_{I \times J} \frac{d y^{\prime} d y_{m}}{\left(1+\left|x_{I}\right|+\left|x_{J}\right|\right)^{M}\left(1+\left|x^{\prime}-x_{I}\right|+\left|x_{m}-x_{J}\right|\right)^{M}} .
\end{aligned}
$$

Note that if $y^{\prime} \in I$ and $y_{m} \in J$, then $\ell(I)+\left|x^{\prime}-x_{I}\right| \sim \ell(I)+\left|x^{\prime}-y^{\prime}\right|, \ell(I)+\left|x_{I}\right| \sim$ $\ell(I)+\left|y^{\prime}\right|, \ell(J)+\left|x_{m}-x_{J}\right| \sim \ell(J)+\left|x_{m}-y_{m}\right|$, and $\ell(J)+\left|x_{J}\right| \sim \ell(J)+\left|y_{m}\right|$. Then simple calculation gives

$$
\begin{aligned}
\frac{1}{\left(1+\left|x^{\prime}-x_{I}\right|+\left|x_{m}-x_{J}\right|\right)^{M}} & \leq \frac{2^{|j| 2 M} 2^{|k| 3 M}}{\left(\ell(I)+\ell(J)+\left|x^{\prime}-x_{I}\right|+\left|x_{m}-x_{J}\right|\right)^{M}} \\
& \leq \frac{2^{|j| 4 M} 2^{|k| 6 M}}{\left(1+\left|x^{\prime}-y^{\prime}\right|+\left|x_{m}-y_{m}\right|\right)^{M}} .
\end{aligned}
$$


Similarly,

$$
\frac{1}{\left(1+\left|x_{I}\right|+\left|x_{J}\right|\right)^{M}} \leq \frac{2^{|j| 4 M} 2^{|k| 6 M}}{\left(1+\left|y^{\prime}\right|+\left|y_{m}\right|\right)^{M}} .
$$

This implies that the last term in (2.9) is dominated by

$$
C 2^{-|k|(L-20 M-2|\alpha|-2)} 2^{-|j|(L-20 M-m-|\alpha|)} \frac{1}{\left(1+\left|x^{\prime}\right|+\left|x_{m}\right|\right)^{M}} .
$$

Choosing $L=20 M+2|\alpha|+m+3$, we derive the estimates in (2.4) and hence that the series in (2.3) converges to zero as $N_{1}$ and $N_{2}$ tend to infinity. Therefore, the series in (1.12) converges in $\mathcal{S}_{0}\left(\mathbb{R}^{m}\right)$. By a duality argument, we obtain that the series in (1.12) converges in $\mathcal{S}_{0}^{\prime}\left(\mathbb{R}^{m}\right)$. The proof of Theorem 1.3 is concluded.

\section{Proof of Theorem 1.6}

In this section, we first derive almost orthogonality estimates in Lemma 3.1 and a discrete version of the maximal estimate in Lemma 3.2. Lemmas 3.1 and 3.2 together with Theorem 1.3 yield Theorem 1.6.

Lemma 3.1 (Almost orthogonality estimates). Suppose that $\psi_{j, k}$ and $\varphi_{j^{\prime}, k^{\prime}}$ satisfy the conditions (1.5)-(1.8). Then for any given integers $L$ and $M$, there exists a constant $C=C(L, M)>0$ such that

$$
\begin{aligned}
\left|\psi_{j, k} * \varphi_{j^{\prime}, k^{\prime}}\left(x^{\prime}, x_{m}\right)\right| \leq C & 2^{-\left|j-j^{\prime}\right| L} 2^{-\left|k-k^{\prime}\right| L} \frac{2^{\left(j \wedge j^{\prime} \wedge k \wedge k^{\prime}\right)(m-1)}}{\left(1+2^{j \wedge j^{\prime} \wedge k \wedge k^{\prime}}\left|x^{\prime}\right|\right)^{(M+m-1)}} \\
& \times \frac{2^{j \wedge j^{\prime} \wedge 2\left(k \wedge k^{\prime}\right)}}{\left(1+2^{j \wedge j^{\prime} \wedge 2\left(k \wedge k^{\prime}\right)}\left|x_{m}\right|\right)^{(M+1)}} .
\end{aligned}
$$

Proof. We first write

$\left(\psi_{j, k} * \varphi_{j^{\prime}, k^{\prime}}\right)\left(x^{\prime}, x_{m}\right)=\int_{\mathbb{R}^{m-1} \times \mathbb{R}}\left(\psi_{j}^{(1)} * \varphi_{j^{\prime}}^{(1)}\right)\left(x^{\prime}-y^{\prime}, x_{m}-y_{m}\right)\left(\psi_{k}^{(2)} * \varphi_{k^{\prime}}^{(2)}\right)\left(y^{\prime}, y_{m}\right) d y^{\prime} d y_{m}$.

By almost orthogonality estimates, as in (2.4) and (2.5), we have

$$
\left|\psi_{j}^{(1)} * \varphi_{j^{\prime}}^{(1)}\left(u^{\prime}, u_{m}\right)\right| \leq C \frac{2^{\left(j \wedge j^{\prime}\right) m} 2^{-\left|j-j^{\prime}\right| L}}{\left(1+2^{\left(j \wedge j^{\prime}\right)}\left|u^{\prime}\right|\right)^{(M+m-1)}\left(1+2^{\left(j \wedge j^{\prime}\right)}\left|u_{m}\right|\right)^{(M+1)}} .
$$

and

$$
\left|\psi_{k}^{(2)} * \varphi_{k^{\prime}}^{(2)}\left(y^{\prime}, y_{m}\right)\right| \leq C \frac{2^{\left(k \wedge k^{\prime}\right)(m+1)} 2^{-\left|k-k^{\prime}\right| L}}{\left(1+2^{\left(k \wedge k^{\prime}\right)}\left|y^{\prime}\right|\right)^{(M+m-1)}\left(1+2^{2\left(k \wedge k^{\prime}\right)}\left|y_{m}\right|\right)^{(M+1)}}
$$

The estimates (3.1) and (3.2) imply that

$$
\left|\left(\psi_{j, k} * \varphi_{j^{\prime}, k^{\prime}}\right)\left(x^{\prime}, x_{m}\right)\right| \leq C 2^{-\left|j-j^{\prime}\right| L} 2^{-\left|k-k^{\prime}\right| L} A B,
$$


where

$$
\begin{aligned}
A & =\int_{\mathbb{R}} \frac{2^{\left(j \wedge j^{\prime}\right)}}{\left(1+2^{\left(j \wedge j^{\prime}\right)}\left|y_{m}\right|\right)^{(M+1)}} \frac{2^{2\left(k \wedge k^{\prime}\right)}}{\left(1+2^{2\left(k \wedge k^{\prime}\right)}\left|x_{m}-y_{m}\right|\right)^{(M+1)}} d y_{m} \\
& \leq C \frac{2^{\left(j \wedge j^{\prime} \wedge k \wedge k^{\prime}\right)(m-1)}}{\left(1+2^{j \wedge j^{\prime} \wedge k \wedge k^{\prime}}\left|x^{\prime}\right|\right)^{(M+m-1)}}
\end{aligned}
$$

and

$$
\begin{aligned}
B & =\int_{\mathbb{R}^{m-1}} \frac{2^{\left(j \wedge j^{\prime}\right)(m-1)}}{\left(1+2^{\left(j \wedge j^{\prime}\right)}\left|y^{\prime}\right|\right)^{(M+m-1)}} \frac{2^{\left(k \wedge k^{\prime}\right)(m-1)}}{\left(1+2^{\left(k \wedge k^{\prime}\right)}\left|x^{\prime}-y^{\prime}\right|\right)^{(M+m-1)}} d y^{\prime} \\
& \leq C \frac{2^{j \wedge j^{\prime} \wedge 2\left(k \wedge k^{\prime}\right)}}{\left(1+2^{j \wedge j^{\prime} \wedge 2\left(k \wedge k^{\prime}\right)}\left|x_{m}\right|\right)^{(M+1)}} .
\end{aligned}
$$

This implies the conclusion of Lemma 3.1.

Now we prove the following estimate of the discrete version of the maximal function.

Lemma 3.2. Let $I$ and $I^{\prime}$ be dyadic cubes in $\mathbb{R}^{m-1}$, and let $J$ and $J^{\prime}$ be dyadic intervals in $\mathbb{R}$ with side lengths $\ell(I)=2^{-(j \wedge k)}, \ell\left(I^{\prime}\right)=2^{-\left(j^{\prime} \wedge k^{\prime}\right)}$ and $\ell(J)=2^{-(j \wedge 2 k)}$, $\ell\left(J^{\prime}\right)=2^{-\left(j^{\prime} \wedge 2 k^{\prime}\right)}$, and let the lower left corners of $I$ and $I^{\prime}$ and the left endpoints of $J$ and $J^{\prime}$ be $2^{-(j \wedge k)} \ell^{\prime}, 2^{-\left(j^{\prime} \wedge k^{\prime}\right)} \ell^{\prime \prime}, 2^{-(j \wedge 2 k)} \ell_{m}$ and $2^{-\left(j^{\prime} \wedge 2 k^{\prime}\right)} \ell_{m}^{\prime}$, respectively. Then, for any $u^{\prime}, v^{\prime} \in I, u_{m}, v_{m} \in J$ and any $\frac{m-1}{M+m-1}<\delta \leq 1$,

$$
\begin{aligned}
& \sum_{\left(\ell^{\prime \prime}, \ell_{m}^{\prime}\right) \in \mathbb{Z}^{m-1} \times \mathbb{Z}} \frac{2^{(m-1)\left(j \wedge j^{\prime} \wedge k \wedge k^{\prime}\right)} 2^{j \wedge j^{\prime} \wedge 2 k \wedge 2 k^{\prime}} 2^{-(m-1)\left(j^{\prime} \wedge k^{\prime}\right)} 2^{-\left(j^{\prime} \wedge 2 k^{\prime}\right)}}{\left(1+2^{j \wedge j^{\prime} \wedge k \wedge k^{\prime}}\left|u^{\prime}-2^{-\left(j^{\prime} \wedge k^{\prime}\right)} \ell^{\prime \prime}\right|\right)^{(M+m-1)}} \\
& \times \frac{\left|\left(\varphi_{j^{\prime}, k^{\prime}} * f\right)\left(2^{-\left(j^{\prime} \wedge k^{\prime}\right)} \ell^{\prime \prime}, 2^{-\left(j^{\prime} \wedge 2 k^{\prime}\right)} \ell_{m}^{\prime}\right)\right|}{\left(1+2^{j \wedge j^{\prime} \wedge 2 k \wedge 2 k^{\prime}}\left|u_{m}-2^{-\left(j^{\prime} \wedge 2 k^{\prime}\right)} \ell_{m}^{\prime}\right|\right)^{(M+1)}} \\
& \leq C_{1}\left\{\mathcal{M}_{s}\left[\left(\sum_{\left(\ell^{\prime \prime}, \ell_{m}^{\prime}\right) \in \mathbb{Z}^{m-1} \times \mathbb{Z}}\left|\left(\varphi_{j^{\prime}}, k^{\prime} * f\right)\left(2^{-\left(j^{\prime} \wedge k^{\prime}\right)} \ell^{\prime \prime}, 2^{-\left(j^{\prime} \wedge 2 k^{\prime}\right)} \ell_{m}^{\prime}\right)\right|^{2} \chi_{I^{\prime}} \chi_{J^{\prime}}\right)^{\frac{\delta}{2}}\right]\right\}^{\frac{1}{\delta}}\left(v^{\prime}, v_{m}\right)
\end{aligned}
$$

where

$$
C_{1}=C 2^{(m-1)(1 / \delta-1)\left(j^{\prime} \wedge k^{\prime}-j \wedge k\right)_{+}} 2^{(1 / \delta-1)\left(j^{\prime} \wedge 2 k^{\prime}-j \wedge 2 k\right)_{+}} .
$$

Here $(a-b)_{+}=\max \{a-b, 0\}$, and $\mathcal{M}_{s}$ is the strong maximal function.

Before proving Lemma 3.2, we would like to point out that this lemma is the key tool in showing Theorems 1.6 and 1.7. The discrete version plays a crucial role for this maximal function estimate. This is why we choose the discrete LittlewoodPaley-Stein square function and use it to define the Hardy space.

Proof of Lemma 3.2. For the sake of convenience, we denote by $x_{I}=2^{-(j \wedge k)} \ell^{\prime}$ and $x_{I^{\prime}}=2^{-\left(j^{\prime} \wedge k^{\prime}\right)} \ell^{\prime \prime}$ the lower left corners of $I$ and $I^{\prime}$, and by $x_{J}=2^{-(j \wedge 2 k)} \ell_{m}$ and $x_{J^{\prime}}=2^{-\left(j^{\prime} \wedge 2 k^{\prime}\right)} \ell_{m}^{\prime}$ the left endpoints of $J$ and $J^{\prime}$, respectively. Set

$$
A_{0}=\left\{I^{\prime}: \frac{\left|u^{\prime}-x_{I^{\prime}}\right|}{2^{-\left(j \wedge j^{\prime} \wedge k \wedge k^{\prime}\right)}} \leq 1\right\}, \quad B_{0}=\left\{J^{\prime}: \frac{\left|u_{m}-x_{J^{\prime}}\right|}{2^{-\left(j \wedge j^{\prime} \wedge 2 k \wedge 2 k^{\prime}\right)}} \leq 1\right\},
$$


and for $r \geq 1$ and $s \geq 1$,

$A_{r}=\left\{I^{\prime}: 2^{r-1}<\frac{\left|u^{\prime}-x_{I^{\prime}}\right|}{2^{-\left(j \wedge j^{\prime} \wedge k \wedge k^{\prime}\right)}} \leq 2^{r}\right\}, \quad B_{s}=\left\{J^{\prime}: 2^{s-1}<\frac{\left|u_{m}-x_{J^{\prime}}\right|}{2^{-\left(j \wedge j^{\prime} \wedge 2 k \wedge 2 k^{\prime}\right)}} \leq 2^{s}\right\}$.

For any fixed $r, s \geq 0$, let

$$
\begin{aligned}
E=\left\{\left(w^{\prime}, w_{m}\right) \in \mathbb{R}^{m-1} \times \mathbb{R}:\right. & \left|w^{\prime}-u^{\prime}\right| \leq 2^{r-\left(j \wedge j^{\prime} \wedge k \wedge k^{\prime}\right)}+2^{-(j \wedge k)}, \\
& \left.\left|w_{m}-u_{m}\right| \leq 2^{r-\left(j \wedge j^{\prime} \wedge 2 k \wedge 2 k^{\prime}\right)}+2^{-(j \wedge 2 k)}\right\} .
\end{aligned}
$$

Then $A_{r} \times B_{s} \subset E$, and for any $\left(v^{\prime}, v_{m}\right) \in I \times J,\left(v^{\prime}, v_{m}\right) \in E$. Obviously,

$$
|E| \leq C 2^{(m-1)\left[r-\left(j \wedge j^{\prime} \wedge k \wedge k^{\prime}\right)\right]} 2^{\left[s-\left(j \wedge j^{\prime} \wedge 2 k \wedge 2 k^{\prime}\right)\right]} .
$$

Thus for $\frac{m-1}{M+m-1}<\delta \leq 1$,

$$
\begin{aligned}
& \sum_{\left(\ell^{\prime \prime}, \ell_{m}^{\prime}\right) \in \mathbb{Z}^{m-1} \times \mathbb{Z}} \frac{2^{(m-1)\left(j \wedge j^{\prime} \wedge k \wedge k^{\prime}\right)} 2^{j \wedge j^{\prime} \wedge 2 k \wedge 2 k^{\prime}} 2^{-(m-1)\left(j^{\prime} \wedge k^{\prime}\right)} 2^{-\left(j^{\prime} \wedge 2 k^{\prime}\right)}}{\left(1+2^{j \wedge j^{\prime} \wedge k \wedge k^{\prime}}\left|u^{\prime}-2^{-\left(j^{\prime} \wedge k^{\prime}\right)} \ell^{\prime \prime}\right|\right)^{(M+m-1)}} \\
& \times \frac{\left|\left(\varphi_{j^{\prime}, k^{\prime}} * f\right)\left(2^{-\left(j^{\prime} \wedge k^{\prime}\right)} \ell^{\prime \prime}, 2^{-\left(j^{\prime} \wedge 2 k^{\prime}\right)} \ell_{m}^{\prime}\right)\right|}{\left(1+2^{j \wedge j^{\prime} \wedge 2 k \wedge 2 k^{\prime}}\left|u_{m}-2^{-\left(j^{\prime} \wedge 2 k^{\prime}\right)} \ell_{m}^{\prime}\right|\right)^{(M+1)}} \\
& \leq C \sum_{r, s \geq 0} 2^{-r(M+m-1)} 2^{-s(M+1)} 2^{(m-1)\left(j \wedge j^{\prime} \wedge k \wedge k^{\prime}\right)} 2^{-(m-1)\left(j^{\prime} \wedge k^{\prime}\right)} 2^{j \wedge j^{\prime} \wedge 2 k \wedge 2 k^{\prime}} \\
& \times 2^{-\left(j^{\prime} \wedge 2 k^{\prime}\right)}\left(\sum_{I^{\prime} \times J^{\prime} \in A_{r} \times B_{s}}\left|\left(\varphi_{j^{\prime}, k^{\prime}} * f\right)\left(x_{I^{\prime}}, x_{J^{\prime}}\right)\right|^{\delta}\right)^{1 / \delta} \\
& =C \sum_{r, s \geq 0} 2^{-r(M+m-1)} 2^{-s(M+1)} 2^{(m-1)\left(j \wedge j^{\prime} \wedge k \wedge k^{\prime}\right)} 2^{j \wedge j^{\prime} \wedge 2 k \wedge 2 k^{\prime}}\left|I^{\prime}\right|\left|J^{\prime}\right||E|^{1 / \delta} \\
& \times\left\{\frac{1}{|E|} \int_{E} \sum_{I^{\prime} \times J^{\prime} \in A_{r} \times B_{s}}\left|I^{\prime}\right|^{-1}\left|J^{\prime}\right|^{-1}\left|\left(\varphi_{j^{\prime}, k^{\prime}} * f\right)\left(x_{I^{\prime}}, x_{J^{\prime}}\right)\right|^{\delta} \chi_{I^{\prime}} \chi_{J^{\prime}} d x\right\}^{1 / \delta} \\
& \leq C \sum_{r, s \geq 0} 2^{-r(M+m-1)} 2^{-s(M+1)} 2^{(m-1)\left(j \wedge j^{\prime} \wedge k \wedge k^{\prime}\right)} 2^{j \wedge j^{\prime} \wedge 2 k \wedge 2 k^{\prime}}\left|I^{\prime}\right|^{1-1 / \delta}\left|J^{\prime}\right|^{1-1 / \delta} \\
& \times|E|^{1 / \delta}\left\{\mathcal{M}_{s}\left(\sum_{I^{\prime} \times J^{\prime} \in A_{r} \times B_{s}}\left|\left(\varphi_{j^{\prime}, k^{\prime}} * f\right)\left(x_{I^{\prime}}, x_{J^{\prime}}\right)\right|^{\delta} \chi_{I^{\prime}} \chi_{J^{\prime}}\right)\left(v^{\prime}, v_{m}\right)\right\}^{1 / \delta} \\
& \leq C_{1}\left\{\mathcal{M}_{s}\left(\sum_{I^{\prime} \times J^{\prime}}\left|\left(\varphi_{j^{\prime}, k^{\prime}} * f\right)\left(x_{I^{\prime}}, x_{J^{\prime}}\right)\right|^{\delta} \chi_{I^{\prime}} \chi_{J^{\prime}}\right)\left(v^{\prime}, v_{m}\right)\right\}^{1 / \delta} \\
& =C_{1}\left\{\mathcal{M}_{s}\left[\left(\sum_{I^{\prime} \times J^{\prime}}\left|\left(\varphi_{j^{\prime}, k^{\prime}} * f\right)\left(x_{I^{\prime}}, x_{J^{\prime}}\right)\right|^{2} \chi_{I^{\prime}} \chi_{J^{\prime}}\right)^{\delta / 2}\right]\left(v^{\prime}, v_{m}\right)\right\}^{1 / \delta} \\
& =C_{1}\left\{\mathcal{M}_{s}\left[\left(\sum_{\left(\ell^{\prime \prime}, \ell_{m}^{\prime}\right) \in \mathbb{Z}^{m-1} \times \mathbb{Z}}\left|\left(\varphi_{j^{\prime}, k^{\prime}} * f\right)\left(2^{-\left(j^{\prime} \wedge k^{\prime}\right)} \ell^{\prime \prime}, 2^{-\left(j^{\prime} \wedge 2 k^{\prime}\right)} \ell_{m}^{\prime}\right)\right|^{2} \chi_{I^{\prime}} \chi_{J^{\prime}}\right)^{\frac{\delta}{2}}\right]\left(v^{\prime}, v_{m}\right)\right\}^{\frac{1}{\delta}} .
\end{aligned}
$$


Now we return to:

Proof of Theorem 1.6. Let $f \in \mathcal{S}_{0}^{\prime}\left(\mathbb{R}^{m}\right)$. We write $x_{I}=2^{-(j \wedge k)} \ell^{\prime}, x_{J}=2^{-(j \wedge 2 k)} \ell_{m}$, $x_{I^{\prime}}=2^{-\left(j^{\prime} \wedge k^{\prime}\right)} \ell^{\prime \prime}$ and $x_{J^{\prime}}=2^{-\left(j^{\prime} \wedge 2 k^{\prime}\right)} \ell_{m}^{\prime}$. The discrete Calderón identity on $\mathcal{S}^{\prime} / \mathcal{P}\left(\mathbb{R}^{m}\right)$ and the almost orthogonality estimates yield that for $\frac{m-1}{M+m-1}<\delta<$ $p \leq 1$ and any $v^{\prime} \in I$ and $v_{m} \in J$,

$$
\begin{aligned}
& \left|\left(\psi_{j, k} * f\right)\left(x_{I}, x_{J}\right)\right| \\
& =\mid \sum_{j^{\prime}, k^{\prime}} \sum_{\left(\ell^{\prime \prime}, \ell_{m}^{\prime}\right)} 2^{-(m-1)\left(j^{\prime} \wedge k^{\prime}\right)} 2^{-\left(j^{\prime} \wedge 2 k^{\prime}\right)} \\
& \times\left(\psi_{j, k} * \varphi_{j^{\prime}, k^{\prime}}\right)\left(x_{I}-x_{I^{\prime}}, x_{J}-x_{J^{\prime}}\right)\left(\varphi_{j^{\prime}, k^{\prime}} * f\right)\left(x_{I^{\prime}}, x_{J^{\prime}}\right) \mid \\
& \leq C \sum_{j^{\prime}, k^{\prime}} 2^{-\left|j-j^{\prime}\right| L} 2^{-\left|k-k^{\prime}\right| L} \\
& \times \sum_{\left(\ell^{\prime \prime}, \ell_{m}^{\prime}\right)} \frac{2^{(m-1)\left(j \wedge j^{\prime} \wedge k \wedge k^{\prime}\right)} 2^{j \wedge j^{\prime} \wedge 2 k \wedge 2 k^{\prime}} 2^{-(m-1)\left(j^{\prime} \wedge k^{\prime}\right)} 2^{-\left(j^{\prime} \wedge 2 k^{\prime}\right)}}{\left(1+2^{j \wedge j^{\prime} \wedge k \wedge k^{\prime}}\left|x_{I}-x_{I^{\prime}}\right|\right)^{(M+m-1)}} \\
& \times \frac{\left|\left(\varphi_{j^{\prime}, k^{\prime}} * f\right)\left(x_{I^{\prime}}, x_{J^{\prime}}\right)\right|}{\left(1+2^{j \wedge j^{\prime} \wedge 2 k \wedge 2 k^{\prime}}\left|x_{J}-x_{J^{\prime}}\right|\right)^{(M+1)}} \\
& \leq C \sum_{j^{\prime}, k^{\prime}} 2^{-\left|j-j^{\prime}\right| L} 2^{-\left|k-k^{\prime}\right| L} \\
& \times C_{1}\left\{\mathcal{M}_{s}\left[\left(\sum_{\left(\ell^{\prime \prime}, \ell_{m}^{\prime}\right)}\left|\left(\varphi_{j^{\prime}, k^{\prime}} * f\right)\left(x_{I^{\prime}}, x_{J^{\prime}}\right)\right|^{2} \chi_{I^{\prime}} \chi_{J^{\prime}}\right)^{\delta / 2}\right]\left(v^{\prime}, v_{m}\right)\right\}^{1 / \delta},
\end{aligned}
$$

where the last inequality follows from Lemma 3.2. Squaring both sides, then multiplying by $\chi_{I}$ and $\chi_{J}$, summing over all $j, k \in \mathbb{Z}$ and $\left(\ell^{\prime}, \ell_{m}\right) \in \mathbb{Z}^{m-1} \times \mathbb{Z}$, and finally applying Hölder's inequality we obtain that for any $x^{\prime} \in I, x_{m} \in J$, and $\max \left\{\frac{m+1}{L+m+1}, \frac{m-1}{M+m-1}\right\}<\delta<p \leq 1$,

$$
\begin{aligned}
& \left|\mathcal{G}_{\psi}^{d}(f)\left(x^{\prime}, x_{m}\right)\right|^{2} \\
& \quad \leq C \sum_{j, k}\left\{\sum_{j^{\prime}, k^{\prime}} 2^{-\left|j-j^{\prime}\right| L} 2^{-\left|k-k^{\prime}\right| L} 2^{(m-1)\left(\frac{1}{\delta}-1\right)\left(j^{\prime} \wedge k^{\prime}-j \wedge k\right)_{+}} 2^{\left(\frac{1}{\delta}-1\right)\left(j^{\prime} \wedge 2 k^{\prime}-j \wedge 2 k\right)_{+}}\right\} \\
& \quad \times\left\{\sum_{j^{\prime}, k^{\prime}} 2^{-\left|j-j^{\prime}\right| L} 2^{-\left|k-k^{\prime}\right| L} 2^{(m-1)\left(\frac{1}{\delta}-1\right)\left(j^{\prime} \wedge k^{\prime}-j \wedge k\right)+} 2^{\left(\frac{1}{\delta}-1\right)\left(j^{\prime} \wedge 2 k^{\prime}-j \wedge 2 k\right)_{+}}\right. \\
& \left.\left.\quad \times\left\{\mathcal{M}_{s}\left[\sum_{\left(\ell^{\prime \prime}, \ell_{m}^{\prime}\right) \in \mathbb{Z}^{m-1} \times \mathbb{Z}}\left|\left(\varphi_{j^{\prime}, k^{\prime}} * f\right)\left(2^{-\left(j^{\prime} \wedge k^{\prime}\right)} \ell^{\prime \prime}, 2^{-\left(j^{\prime} \wedge 2 k^{\prime}\right)} \ell_{m}^{\prime}\right)\right|^{2} \chi_{I^{\prime}} \chi_{J^{\prime}}\right)^{\delta / 2}\right]\left(x^{\prime}, x_{m}\right)\right\}^{2 / \delta}\right\} \\
& \leq C\left\{\sum _ { j ^ { \prime } , k ^ { \prime } } \left\{\mathcal { M } _ { s } \left[\left(\sum_{\left(\ell^{\prime \prime}, \ell_{m}^{\prime}\right) \in \mathbb{Z}^{m-1} \times \mathbb{Z}}\left|\left(\varphi_{j^{\prime}, k^{\prime}} * f\right)\left(2^{-\left(j^{\prime} \wedge k^{\prime}\right)} \ell^{\prime \prime}, 2^{-\left(j^{\prime} \wedge 2 k^{\prime}\right)} \ell_{m}^{\prime}\right)\right|^{2}\right.\right.\right.\right. \\
& \left.\left.\left.\left.\quad \times \chi_{I^{\prime}} \chi_{J^{\prime}}\right)^{\delta / 2}\right]\left(x^{\prime}, x_{m}\right)\right\}^{2 / \delta}\right\},
\end{aligned}
$$

where in the last inequality we use the facts that $\left(j^{\prime} \wedge k^{\prime}-j \wedge k\right)_{+} \leq\left|j-j^{\prime}\right|$ $+\left|k-k^{\prime}\right|,\left(j^{\prime} \wedge 2 k^{\prime}-j \wedge 2 k\right)_{+} \leq\left|j-j^{\prime}\right|+2\left|k-k^{\prime}\right|$, and that if $L$ is chosen so 
that $L>(m+1)(1 / \delta-1)$, then

$$
\sum_{j^{\prime}, k^{\prime}} 2^{-\left|j-j^{\prime}\right| L} 2^{-\left|k-k^{\prime}\right| L} 2^{(m-1)(1 / \delta-1)\left(j^{\prime} \wedge k^{\prime}-j \wedge k\right)+} 2^{(1 / \delta-1)\left(j^{\prime} \wedge 2 k^{\prime}-j \wedge 2 k\right)+} \leq C
$$

and

$$
\sum_{j, k} 2^{-\left|j-j^{\prime}\right| L} 2^{-\left|k-k^{\prime}\right| L} 2^{(m-1)(1 / \delta-1)\left(j^{\prime} \wedge k^{\prime}-j \wedge k\right)+} 2^{(1 / \delta-1)\left(j^{\prime} \wedge 2 k^{\prime}-j \wedge 2 k\right)+} \leq C .
$$

Applying Fefferman-Stein's vector-valued strong maximal inequality on $L^{p / \delta}\left(\ell^{2 / \delta}\right)$ yields

$$
\left\|\mathcal{G}_{\psi}^{d}(f)\right\|_{L^{p}\left(\mathbb{R}^{m}\right)} \leq C\left\|\mathcal{G}_{\varphi}^{d}(f)\right\|_{L^{p}\left(\mathbb{R}^{m}\right)} .
$$

The conclusion of Theorem 1.6 follows.

As a consequence of Theorem 1.6, $L^{2}\left(\mathbb{R}^{m}\right) \cap H_{\text {com }}^{p}\left(\mathbb{R}^{m}\right)$ is dense in $H_{\text {com }}^{p}\left(\mathbb{R}^{m}\right)$. Indeed we have the following result:

Corollary 3.3. $\mathcal{S}_{0}\left(\mathbb{R}^{m}\right)$ is dense in $H_{\mathrm{com}}^{p}\left(\mathbb{R}^{m}\right)$.

Proof. Let $f \in H_{\text {com }}^{p}\left(\mathbb{R}^{m}\right)$. For any fixed $N>0$, let

$$
E=\left\{\left(j, k, \ell^{\prime}, \ell_{m}\right):|j| \leq N,|k| \leq N,\left|\ell^{\prime}\right| \leq N,\left|\ell_{m}\right| \leq N\right\},
$$

and

$$
\begin{gathered}
f_{N}\left(x^{\prime}, x_{m}\right):=\sum_{\left(j, k, \ell^{\prime}, \ell_{m}\right) \in E} 2^{-(m-1)(j \wedge k)} 2^{-(j \wedge 2 k)}\left(\psi_{j, k} * f\right)\left(2^{-(j \wedge k)} \ell^{\prime}, 2^{-(j \wedge 2 k)} \ell_{m}\right) \\
\times \psi_{j, k}\left(x^{\prime}-2^{-(j \wedge k)} \ell^{\prime}, x_{m}-2^{-(j \wedge 2 k)} \ell_{m}\right),
\end{gathered}
$$

where $\psi_{j, k}$ is the same as in Theorem 1.3.

Since $\psi_{j, k} \in \mathcal{S}_{0}\left(\mathbb{R}^{m}\right)$, we obviously have $f_{N} \in \mathcal{S}_{0}\left(\mathbb{R}^{m}\right)$. Repeating the proof of Theorem 1.6, we can conclude that $\left\|f_{N}\right\|_{H_{\text {com }}^{p}\left(\mathbb{R}^{m}\right)} \leq C\|f\|_{H_{\text {com }}^{p}\left(\mathbb{R}^{m}\right)}$. To see that $f_{N}$ tends to $f$ in $H_{\text {com }}^{p}$, by the discrete Calderón identity in $\mathcal{S}_{0}^{\prime}\left(\mathbb{R}^{m}\right)$ from Theorem 1.3 ,

$$
\begin{gathered}
\left(f-f_{N}\right)\left(x^{\prime}, x_{m}\right)=\sum_{\left(j, k, \ell^{\prime}, \ell_{m}\right) \in E^{c}} 2^{-(m-1)(j \wedge k)} 2^{-(j \wedge 2 k)}\left(\psi_{j, k} * f\right)\left(2^{-(j \wedge k)} \ell^{\prime}, 2^{-(j \wedge 2 k)} \ell_{m}\right) \\
\times \psi_{j, k}\left(x^{\prime}-2^{-(j \wedge k)} \ell^{\prime}, x_{m}-2^{-(j \wedge 2 k)} \ell_{m}\right),
\end{gathered}
$$

where the series converges in $\mathcal{S}_{0}^{\prime}\left(\mathbb{R}^{m}\right)$. Therefore,

$$
\begin{aligned}
\mathcal{G}_{\psi}( & \left.f-f_{N}\right):=\left\{\sum_{j^{\prime}, k^{\prime}} \sum_{\left(\ell^{\prime \prime}, \ell_{m}\right)}\left|\psi_{j^{\prime}, k^{\prime}} *\left(f-f_{N}\right)\left(2^{-\left(j^{\prime} \wedge k^{\prime}\right)} \ell^{\prime \prime}, 2^{-\left(j^{\prime} \wedge 2 k^{\prime}\right)} \ell_{m}^{\prime}\right)\right|^{2} \chi_{I^{\prime}} \chi_{J^{\prime}}\right\}^{1 / 2} \\
= & \left\{\sum_{j^{\prime}, k^{\prime}} \sum_{\left(\ell^{\prime \prime}, \ell_{m}\right)} \mid \psi_{j^{\prime}, k^{\prime}} * \sum_{\left(j, k, \ell^{\prime}, \ell_{m}\right) \in E^{c}} 2^{-(m-1)(j \wedge k)} 2^{-(j \wedge 2 k)}\right. \\
& \times\left(\psi_{j, k} * f\right)\left(2^{-(j \wedge k)} \ell^{\prime}, 2^{-(j \wedge 2 k)} \ell_{m}\right) \\
& \left.\times\left.\psi_{j^{\prime}, k^{\prime}} * \psi_{j, k}\left(2^{-\left(j^{\prime} \wedge k^{\prime}\right)} \ell^{\prime \prime}-2^{-(j \wedge k)} \ell^{\prime}, 2^{-\left(j^{\prime} \wedge 2 k^{\prime}\right)} \ell_{m}^{\prime}-2^{-(j \wedge 2 k)} \ell_{m}\right)\right|^{2} \chi_{I^{\prime}} \chi_{J^{\prime}}\right\}^{1 / 2} .
\end{aligned}
$$


Repeating the proof of Theorem 1.6 yields

$$
\begin{aligned}
& \left\|\mathcal{G}_{\varphi}\left(f-f_{N}\right)\right\|_{L^{p}\left(\mathbb{R}^{m}\right)} \\
& \quad \leq C\left\|\left\{\sum_{\left(j, k, \ell^{\prime}, \ell_{m}\right) \in E^{c}}\left|\psi_{j, k} * f\left(2^{-(j \wedge k)} \ell^{\prime}, 2^{-(j \wedge 2 k)} \ell_{m}\right)\right|^{2} \chi_{I} \chi_{J}\right\}^{1 / 2}\right\|_{L^{p}\left(\mathbb{R}^{m}\right)},
\end{aligned}
$$

where the last term tends to 0 as $N$ tends to infinity. This implies that $f_{N}$ tends to $f$ in the $H_{\text {com }}^{p}\left(\mathbb{R}^{m}\right)$ norm as $N$ tend to infinity.

\section{Proof of Theorem 1.7}

To show Theorem 1.7, we need a discrete Calderón-type identity on $L^{2}\left(\mathbb{R}^{m}\right) \cap$ $H_{\text {com }}^{p}\left(\mathbb{R}^{m}\right)$, which has interest on its own. To do this, let $\phi^{(1)} \in \mathcal{S}\left(\mathbb{R}^{m}\right)$ with supp $\phi^{(1)} \subseteq B(0,1)$,

$$
\sum_{j \in \mathbb{Z}}\left|\widehat{\phi^{(1)}}\left(2^{-j} \xi\right)\right|^{2}=1 \quad \text { for all } \xi \in \mathbb{R}^{m} \backslash\{0\}
$$

and

$$
\int_{\mathbb{R}^{m}} \phi^{(1)}(x) x^{\alpha} d x=0 \text { for all }|\alpha| \leq 10 M
$$

where $M$ is a fixed large positive integer depending on $p$. We also let $\phi^{(2)} \in \mathcal{S}\left(\mathbb{R}^{m}\right)$ with supp $\phi^{(2)} \subseteq B(0,1)$,

$$
\sum_{k \in \mathbb{Z}}\left|\widehat{\phi^{(2)}}\left(2^{-k} \xi^{\prime}, 2^{-2 k} \xi_{m}\right)\right|^{2}=1 \quad \text { for all }\left(\xi^{\prime}, \xi_{m}\right) \in \mathbb{R}^{m-1} \times \mathbb{R} \backslash\{(0,0)\},
$$

and

$$
\int_{\mathbb{R}^{m}} \phi^{(2)}(x) x^{\beta} d x=0 \quad \text { for all }|\beta| \leq 10 M .
$$

Set $\phi_{j, k}=\phi_{j}^{(1)} * \phi_{k}^{(2)}$, where

$$
\phi_{j}^{(1)}(x)=2^{j m} \phi^{(1)}\left(2^{j} x\right) \text { and } \phi_{k}^{(2)}\left(x^{\prime}, x_{m}\right)=2^{k(m+1)} \phi^{(2)}\left(2^{k} x^{\prime}, 2^{2 k} x_{m}\right) .
$$

The discrete Calderón-type identity is given by the following:

Theorem 4.1. Let $\phi^{(1)}$ and $\phi^{(2)}$ satisfy conditions (4.1)-(4.4). Then for any $f \in L^{2}\left(\mathbb{R}^{m}\right) \cap H_{\text {com }}^{p}\left(\mathbb{R}^{m}\right)$, there exists $h \in L^{2}\left(\mathbb{R}^{m}\right) \cap H_{\text {com }}^{p}\left(\mathbb{R}^{m}\right)$ such that for a sufficiently large $N \in \mathbb{N}$,

$$
f\left(x^{\prime}, x_{m}\right)=\sum_{j, k \in \mathbb{Z}} \sum_{\left(\ell^{\prime}, \ell_{m}\right) \in \mathbb{Z}^{m-1} \times \mathbb{Z}}|I||J| \phi_{j, k}\left(x^{\prime}-2^{-(j \wedge k)-N} \ell^{\prime}, x_{m}-2^{-(j \wedge 2 k)-N} \ell_{m}\right)
$$

$$
\times\left(\phi_{j, k} * h\right)\left(2^{-(j \wedge k)-N} \ell^{\prime}, 2^{-(j \wedge 2 k)-N} \ell_{m}\right),
$$


where the series converges in $L^{2}$, the $I$ are dyadic cubes in $\mathbb{R}^{m-1}$, and the $J$ are dyadic intervals in $\mathbb{R}$ with side lengths $\ell(I)=2^{-(j \wedge k)-N}$ and $\ell(J)=2^{-(j \wedge 2 k)-N}$, and the lower left corners of $I$ and the left endpoints of $J$ are $2^{-(j \wedge k)-N} \ell^{\prime}$ and $2^{-(j \wedge 2 k)-N} \ell_{m}$, respectively. Moreover,

$$
\|f\|_{L^{2}\left(\mathbb{R}^{m}\right)} \approx\|h\|_{L^{2}\left(\mathbb{R}^{m}\right)},
$$

and

$$
\|f\|_{H_{\mathrm{com}}^{p}\left(\mathbb{R}^{m}\right)} \approx\|h\|_{H_{\mathrm{com}}^{p}\left(\mathbb{R}^{m}\right)} .
$$

We point out that the main difference between the discrete Calderón-type identity above and the discrete Calderón identity given in Theorem 1.3 is that for any fixed $j, k \in \mathbb{Z}$, the $\phi_{j, k}\left(x^{\prime}, x_{m}\right)$ in (4.5) have compact supports but the $\psi_{j, k}\left(x^{\prime}, x_{m}\right)$ in (1.12) do not. Being of compact support allows to use the orthogonality argument in the proof of Theorem 1.7.

Proof of Theorem 4.1. By taking the Fourier transform, we have that for any $f \in$ $L^{2}\left(\mathbb{R}^{m}\right)$,

$$
f\left(x^{\prime}, x_{m}\right)=\sum_{j, k} \phi_{j, k} * \phi_{j, k} * f\left(x^{\prime}, x_{m}\right) .
$$

Applying Coifman's decomposition of the identity operator, we obtain

$$
\begin{aligned}
f\left(x^{\prime}, x_{m}\right)= & \sum_{j, k} \sum_{\left(\ell^{\prime}, \ell_{m}\right)}|I||J| \phi_{j, k}\left(x^{\prime}-2^{-(j \wedge k)-N} \ell^{\prime}, x_{m}-2^{-(j \wedge 2 k)-N} \ell_{m}\right) \\
& \times\left(\phi_{j, k} * f\right)\left(2^{-(j \wedge k)-N} \ell^{\prime}, 2^{-(j \wedge 2 k)-N} \ell_{m}\right)+\mathcal{R}_{N}(f)\left(x^{\prime}, x_{m}\right) \\
:= & T_{N}(f)\left(x^{\prime}, x_{m}\right)+\mathcal{R}_{N}(f)\left(x^{\prime}, x_{m}\right),
\end{aligned}
$$

where

$$
\begin{aligned}
& \mathcal{R}_{N}(f)\left(x^{\prime}, x_{m}\right) \\
& =\sum_{j, k} \sum_{\left(\ell^{\prime}, \ell_{m}\right)} \int_{I \times J}\left[\phi_{j, k}\left(x^{\prime}-y^{\prime}, x_{m}-y_{m}\right)\left(\phi_{j, k} * f\right)\left(y^{\prime}, y_{m}\right)\right. \\
& -\phi_{j, k}\left(x^{\prime}-2^{-(j \wedge k)-N} \ell^{\prime}, x_{m}-2^{-(j \wedge 2 k)-N} \ell_{m}\right) \\
& \left.\times\left(\phi_{j, k} * f\right)\left(2^{-(j \wedge k)-N} \ell^{\prime}, 2^{-(j \wedge 2 k)-N} \ell_{m}\right)\right] d y^{\prime} d y_{m}
\end{aligned}
$$


Here, the $I$ are dyadic cubes in $\mathbb{R}^{m-1}$, and the $J$ are dyadic intervals in $\mathbb{R}$ with side lengths $\ell(I)=2^{-(j \wedge k)-N}$ and $\ell(J)=2^{-(j \wedge 2 k)-N}$ and the lower left corners of $I$ and the left endpoints of $J$ are $2^{-(j \wedge k)-N} \ell^{\prime}$ and $2^{-(j \wedge 2 k)-N} \ell_{m}$, respectively. We claim that for $i=1,2$,

$$
\left\|\mathcal{R}_{N}^{i}(f)\right\|_{L^{2}\left(\mathbb{R}^{m}\right)} \leq C 2^{-N}\|f\|_{L^{2}\left(\mathbb{R}^{m}\right)}
$$

and

$$
\left\|\mathcal{R}_{N}^{i}(f)\right\|_{H_{\mathrm{com}}^{p}\left(\mathbb{R}^{m}\right)} \leq C 2^{-N}\|f\|_{H_{\mathrm{com}}^{p}\left(\mathbb{R}^{m}\right)}
$$

where $C$ is a constant independent of $f$ and $N$.

Assuming the claim for the moment, then, by choosing sufficiently large $N$, $T_{N}^{-1}=\sum_{n=0}^{\infty}\left(\mathcal{R}_{N}\right)^{n}$ is bounded in both $L^{2}$ and $H_{\text {com }}^{p}$, which implies that

$$
\left\|T_{N}^{-1}(f)\right\|_{L^{2}\left(\mathbb{R}^{m}\right)} \approx\|f\|_{L^{2}\left(\mathbb{R}^{m}\right)} \quad \text { and } \quad\left\|T_{N}^{-1}(f)\right\|_{H_{\mathrm{com}}^{p}\left(\mathbb{R}^{m}\right)} \approx\|f\|_{H_{\mathrm{com}}^{p}\left(\mathbb{R}^{m}\right)} .
$$

Moreover, for any $f \in L^{2}\left(\mathbb{R}^{m}\right) \cap H_{\text {com }}^{p}\left(\mathbb{R}^{m}\right)$, set $h=T_{N}^{-1}(f)$. Then

$$
\begin{aligned}
f\left(x^{\prime}, x_{m}\right)= & T_{N}\left(T_{N}^{-1}(f)\right)\left(x^{\prime}, x_{m}\right) \\
=\sum_{j, k \in \mathbb{Z}} \sum_{\left(\ell^{\prime}, \ell_{m}\right) \in \mathbb{Z}^{m-1} \times \mathbb{Z}}|I||J| \phi_{j, k}( & \left.x^{\prime}-2^{-(j \wedge k)-N} \ell^{\prime}, x_{m}-2^{-(j \wedge 2 k)-N} \ell_{m}\right) \\
& \times\left(\phi_{j, k} * h\right)\left(2^{-(j \wedge k)-N} \ell^{\prime}, 2^{-(j \wedge 2 k)-N} \ell_{m}\right),
\end{aligned}
$$

where the series converges in $L^{2}$.

Now we show the claim. Since the proofs for $\mathcal{R}_{N}^{1}$ and $\mathcal{R}_{N}^{2}$ are similar, we only give the proof for $\mathcal{R}_{N}^{1}$. Roughly speaking, the proof is similar to Theorem 1.6. To see this, let $f \in L^{2}\left(\mathbb{R}^{m}\right) \cap H_{\text {com }}^{p}\left(\mathbb{R}^{m}\right)$. Applying the discrete Calderón identity in $L^{2}\left(\mathbb{R}^{m}\right)$ from Theorem 1.3 yields

$$
\begin{aligned}
\psi_{j^{\prime}, k^{\prime}} & * \mathcal{R}_{N}^{1}(f)\left(x^{\prime}, x_{m}\right) \\
= & \sum_{j, k \in \mathbb{Z}} \sum_{\left(\ell^{\prime}, \ell_{m}\right) \in \mathbb{Z}^{m-1} \times \mathbb{Z}} \int_{I \times J} \psi_{j^{\prime}, k^{\prime}} *\left[\phi_{j, k}\left(\cdot-y^{\prime}, \cdot-y_{m}\right)\right. \\
& \left.-\phi_{j, k}\left(\cdot-2^{-(j \wedge k)-N} \ell^{\prime}, \cdot-2^{-(j \wedge 2 k)-N} \ell_{m}\right)\right]\left(x^{\prime}, x_{m}\right)\left(\phi_{j, k} * f\right)\left(y^{\prime}, y_{m}\right) d y^{\prime} d y_{m} \\
= & \sum_{j, k \in \mathbb{Z}\left(\ell^{\prime}, \ell_{m}\right) \in \mathbb{Z}^{m-1} \times \mathbb{Z}} \int_{I \times J} \psi_{j^{\prime}, k^{\prime} *\left[\phi_{j, k}\left(\cdot-y^{\prime}, \cdot-y_{m}\right)\right.} \\
& \left.-\phi_{j, k}\left(\cdot-2^{-(j \wedge k)-N} \ell^{\prime}, \cdot-2^{-(j \wedge 2 k)-N} \ell_{m}\right)\right]\left(x^{\prime}, x_{m}\right) \\
& \times \phi_{j, k} *\left\{\sum_{j^{\prime \prime}, k^{\prime \prime} \in \mathbb{Z}} \sum_{\left(\ell^{\prime \prime \prime}, \ell_{m}^{\prime \prime}\right) \in \mathbb{Z}^{m-1} \times \mathbb{Z}}\left|I^{\prime \prime} \| J^{\prime \prime}\right| \psi_{j^{\prime \prime}, k^{\prime \prime}}\left(\cdot-2^{-\left(j^{\prime \prime} \wedge k^{\prime \prime}\right)} \ell^{\prime \prime \prime}, \cdot-2^{-\left(j^{\prime \prime} \wedge 2 k^{\prime \prime}\right)} \ell_{m}^{\prime \prime}\right)\right. \\
(4.10) & \left.\left(\psi_{j^{\prime \prime}, k^{\prime \prime}} * f\right)\left(2^{-\left(j^{\prime \prime} \wedge k^{\prime \prime}\right)} \ell^{\prime \prime \prime}, 2^{-\left(j^{\prime \prime} \wedge 2 k^{\prime \prime}\right)} \ell_{m}^{\prime \prime}\right)\right\}\left(y^{\prime}, y_{m}\right) d y^{\prime} d y_{m},
\end{aligned}
$$

where the $I^{\prime \prime}$ are dyadic cubes in $\mathbb{R}^{m-1}$ with side length $\ell\left(I^{\prime \prime}\right)=2^{-\left(j^{\prime \prime} \wedge k^{\prime \prime}\right)}$, the $J^{\prime \prime}$ are dyadic intervals in $\mathbb{R}$ with side length $\ell\left(J^{\prime \prime}\right)=2^{-\left(j^{\prime \prime} \wedge 2 k^{\prime \prime}\right)}$, and the lower left corners of $I^{\prime \prime}$ and the left endpoints of $J^{\prime \prime}$ are $2^{-\left(j^{\prime \prime} \wedge k^{\prime \prime}\right)} \ell^{\prime \prime \prime}$ and $2^{-\left(j^{\prime \prime} \wedge 2 k^{\prime \prime}\right)} \ell_{m}^{\prime \prime}$, respectively. 
Set

$$
\widetilde{\phi}_{j, k}=\phi_{j, k}\left(z^{\prime}-y^{\prime}, z_{m}-y_{m}\right)-\phi_{j, k}\left(z^{\prime}-2^{-(j \wedge k)-N} \ell^{\prime}, z_{m}-2^{-(j \wedge 2 k)-N} \ell_{m}\right) .
$$

Then by the almost orthogonality arguments in the proof of Lemma 3.1, we obtain

$$
\begin{aligned}
& \left|\psi_{j^{\prime}, k^{\prime}} * \widetilde{\phi}_{j, k}\left(x^{\prime}, x_{m}\right)\right| \lesssim 2^{-N} 2^{-10 M\left|j-j^{\prime}\right|} 2^{-10 M\left|k-k^{\prime}\right|} \frac{2^{\left(j^{\prime} \wedge k^{\prime}\right)(m-1)}}{\left(1+2^{j^{\prime} \wedge k^{\prime}}\left|x^{\prime}-y^{\prime}\right|\right)^{(M+m-1)}} \\
& \times \frac{2^{j^{\prime} \wedge 2 k^{\prime}}}{\left(1+2^{j^{\prime} \wedge 2 k^{\prime}}\left|x_{m}-y_{m}\right|\right)^{(M+1)}}
\end{aligned}
$$

and similarly, for $y^{\prime} \in I, y_{m} \in J$,

$$
\begin{aligned}
\left|\phi_{j, k} * \psi_{j^{\prime \prime}, k^{\prime \prime}}\left(y^{\prime}-2^{-\left(j^{\prime \prime} \wedge k^{\prime \prime}\right)} \ell^{\prime \prime}, y_{m}-2^{-\left(j^{\prime \prime} \wedge 2 k^{\prime \prime}\right)} \ell_{m}^{\prime}\right)\right| & \\
\lesssim 2^{-10 M\left|j-j^{\prime \prime}\right|} 2^{-10 M\left|k-k^{\prime \prime}\right|} & \frac{2^{\left(j^{\prime \prime} \wedge k^{\prime \prime}\right)(m-1)}}{\left(1+2^{j^{\prime \prime} \wedge k^{\prime \prime}} \mid y^{\prime}-2^{\left.-\left(j^{\prime \prime} \wedge k^{\prime \prime}\right) \ell^{\prime \prime} \mid\right)^{(M+m-1)}}\right.} \\
& \times \frac{2^{j^{\prime \prime} \wedge 2 k^{\prime \prime}}}{\left.\left(1+2^{j^{\prime \prime} \wedge 2 k^{\prime \prime}} \mid y_{m}-2^{-\left(j^{\prime \prime} \wedge 2 k^{\prime \prime}\right)} \ell_{m}^{\prime}\right) \mid\right)^{(M+1)}} .
\end{aligned}
$$

Substituting these estimates into the last term in (4.10) yields

$$
\begin{aligned}
& \left|\psi_{j^{\prime}, k^{\prime}} * \mathbb{R}_{N}^{1}(f)\left(x^{\prime}, x_{m}\right)\right| \\
& \lesssim \sum_{j^{\prime \prime}, k^{\prime \prime} \in \mathbb{Z}} \sum_{\left(\ell^{\prime \prime \prime}, \ell_{m}^{\prime \prime}\right) \in \mathbb{Z}^{m-1} \times \mathbb{Z}}\left|I^{\prime \prime}\left\|J^{\prime \prime}\right\|\left(\psi_{j^{\prime \prime}, k^{\prime \prime}} * f\right)\left(2^{-\left(j^{\prime \prime} \wedge k^{\prime \prime}\right)} \ell^{\prime \prime \prime}, 2^{-\left(j^{\prime \prime} \wedge 2 k^{\prime \prime}\right)} \ell_{m}^{\prime \prime}\right)\right| \\
& \times \sum_{j, k \in \mathbb{Z}} \sum_{\left(\ell^{\prime}, \ell_{m}\right) \in \mathbb{Z}^{m-1} \times \mathbb{Z}} \int_{I \times J} 2^{-N} 2^{-\left|j-j^{\prime}\right| 3 M} 2^{-\left|k-k^{\prime}\right| 3 M} \\
& \times \frac{2^{\left(j^{\prime} \wedge k^{\prime}\right)(m-1)}}{\left(1+2^{j^{\prime} \wedge k^{\prime}}\left|x^{\prime}-y^{\prime}\right|\right)^{(M+m-1)}} \frac{2^{j^{\prime} \wedge 2 k^{\prime}}}{\left(1+2^{j^{\prime} \wedge 2 k^{\prime}}\left|x_{m}-y_{m}\right|\right)^{(M+1)}} \\
& \times 2^{-\left|j-j^{\prime \prime}\right| 3 M} 2^{-\left|k-k^{\prime \prime}\right| 3 M} \frac{2^{\left(j^{\prime \prime} \wedge k^{\prime \prime}\right)(m-1)}}{\left(1+2^{j^{\prime \prime} \wedge k^{\prime \prime}}\left|y^{\prime}-2^{-\left(j^{\prime \prime} \wedge k^{\prime \prime}\right)} \ell^{\prime \prime}\right|\right)^{(M+m-1)}} \\
& \times \frac{2^{j^{\prime \prime} \wedge 2 k^{\prime \prime}}}{\left.\left(1+2^{j^{\prime \prime} \wedge 2 k^{\prime \prime}} \mid y_{m}-2^{-\left(j^{\prime \prime} \wedge 2 k^{\prime \prime}\right)} \ell_{m}^{\prime}\right) \mid\right)^{(M+1)}} d y^{\prime} d y_{m} \\
& \lesssim 2^{-N} \sum_{j^{\prime \prime}, k^{\prime \prime}} \sum_{\left(\ell^{\prime \prime \prime}, \ell_{m}^{\prime \prime}\right)} 2^{-\left|j^{\prime}-j^{\prime \prime}\right| 3 M} 2^{-\left|k^{\prime}-k^{\prime \prime}\right| 3 M}\left|I^{\prime \prime}\right|\left|J^{\prime \prime}\right| \\
& \times \frac{2^{\left(j^{\prime} \wedge j^{\prime \prime} \wedge k^{\prime} \wedge k^{\prime \prime}\right)(m-1)}}{\left(1+2^{j^{\prime} \wedge j^{\prime \prime} \wedge k^{\prime} \wedge k^{\prime \prime}}\left|x^{\prime}-2^{-\left(j^{\prime \prime} \wedge k^{\prime \prime}\right) \ell^{\prime \prime \prime}}\right|\right)^{(M+m-1)}} \\
& \times \frac{2^{\left(j^{\prime} \wedge j^{\prime \prime}\right) \wedge 2\left(k \wedge k^{\prime}\right)}}{\left(1+2^{\left(j^{\prime} \wedge j^{\prime \prime}\right) \wedge 2\left(k \wedge k^{\prime}\right)}\left|x_{m}-2^{-\left(j^{\prime \prime} \wedge 2 k^{\prime \prime}\right)} \ell_{m}^{\prime \prime}\right|\right)^{(M+1)}} \\
& \times\left|\left(\psi_{j^{\prime \prime}, k^{\prime \prime}} * f\right)\left(2^{-\left(j^{\prime \prime} \wedge k^{\prime \prime}\right)} \ell^{\prime \prime \prime}, 2^{-\left(j^{\prime \prime} \wedge 2 k^{\prime \prime}\right)} \ell_{m}^{\prime \prime}\right)\right| \text {. }
\end{aligned}
$$


By the $L^{2}$ boundedness of the discrete Littlewood-Paley-Stein square function $\mathcal{G}_{\psi}^{d}(f)$, we have

$$
\begin{aligned}
& \left\|\mathcal{R}_{N}^{1}(f)\right\|_{L^{2}} \lesssim\left\|\mathcal{G}_{\psi}^{d}\left(\mathcal{R}_{N}^{1} f\right)\left(x^{\prime}, x_{m}\right)\right\|_{L^{2}} \\
& \lesssim 2^{-N}\left\|\left\{\sum_{j^{\prime \prime}, k^{\prime \prime} \in \mathbb{Z}} \sum_{\left(\ell^{\prime \prime \prime}, \ell_{m}^{\prime \prime}\right) \in \mathbb{Z}^{m-1} \times \mathbb{Z}}\left|\left(\psi_{j^{\prime \prime}, k^{\prime \prime}} * f\right)\left(2^{-\left(j^{\prime \prime} \wedge k^{\prime \prime}\right)} \ell^{\prime \prime \prime}, 2^{-\left(j^{\prime \prime} \wedge 2 k^{\prime \prime}\right)} \ell_{m}^{\prime \prime}\right)\right|^{2} \chi_{I}^{\prime \prime} \chi_{J}^{\prime \prime}\right\}^{1 / 2}\right\|_{L^{2}} \\
& \lesssim 2^{-N}\|f\|_{L^{2}} .
\end{aligned}
$$

Repeating the proof, as in Theorem 1.6, implies

$$
\begin{aligned}
& \left\|\mathbb{R}_{N}^{1}(f)\right\|_{H_{\mathrm{com}}^{p}} \lesssim\left\|\mathcal{G}_{\psi}^{d}\left(\mathcal{R}_{N}^{1} f\right)\left(x^{\prime}, x_{m}\right)\right\|_{L^{p}} \\
& \lesssim 2^{-N}\left\|\left\{\sum_{j^{\prime \prime}, k^{\prime \prime} \in \mathbb{Z}} \sum_{\left(\ell^{\prime \prime \prime}, \ell_{m}^{\prime \prime}\right) \in \mathbb{Z}^{m-1} \times \mathbb{Z}}\left|\left(\psi_{j^{\prime \prime}, k^{\prime \prime}} * f\right)\left(2^{-\left(j^{\prime \prime} \wedge k^{\prime \prime}\right)} \ell^{\prime \prime \prime}, 2^{-\left(j^{\prime \prime} \wedge 2 k^{\prime \prime}\right)} \ell_{m}^{\prime \prime}\right)\right|^{2} \chi_{I}^{\prime \prime} \chi_{J}^{\prime \prime}\right\}^{1 / 2}\right\|_{L^{p}} \\
& \lesssim 2^{-N}\|f\|_{H_{\mathrm{com}}^{p}} .
\end{aligned}
$$

The claim is concluded and hence Theorem 4.1 follows.

Arguing as in the proof of Theorem 1.6, we deduce, from Theorem 4.1:

Corollary 4.2. Let $0<p \leq 1$. Suppose $\phi_{j, k}$ satisfies the same conditions as in Theorem 4.1 with a large $M$ depending on $p$. Then for a large $N$ as in Theorem 4.1 and $f \in L^{2} \cap H_{\text {com }}^{p}$,

$$
\|f\|_{H_{\mathrm{com}}^{p}} \approx\left\|\left(\sum_{j, k \in \mathbb{Z}} \sum_{\left(\ell^{\prime}, \ell_{m}\right) \in \mathbb{Z}^{m-1} \times \mathbb{Z}}\left|\left(\phi_{j, k} * f\right)\left(2^{-(j \wedge k)-N} \ell^{\prime}, 2^{-(j \wedge 2 k)-N} \ell_{m}\right)\right|^{2} \chi_{I} \chi_{J}\right)^{1 / 2}\right\|_{L^{p}} .
$$

We now prove Theorem 1.7.

Proof of Theorem 1.7. We may assume that $\mathcal{K}_{i}$ is the kernel of the convolution operator $T_{i}, i=1,2$, and $\mathcal{K}$ is the kernel of the composition operator $T=T_{1} \circ T_{2}$. Then $T(f)=\mathcal{K} * f$ and $\mathcal{K}=\mathcal{K}_{1} * \mathcal{K}_{2}$. For $f \in L^{2} \cap H_{\text {com }}^{p}, 0<p \leq 1$, by the $L^{2}$ boundedness of $T$ and the discrete Calderón identity of Theorem 4.1 applied to $f$ in $L^{2} \cap H_{\text {com }}^{p}$, we conclude

$$
\begin{aligned}
& \|T(f)\|_{H_{\mathrm{com}}^{p}} \\
& \leq C\left\|\left\{\sum_{j, k} \sum_{\left(\ell^{\prime}, \ell_{m}\right)}\left|\left(\phi_{j, k} * \mathcal{K} * f\right)\left(2^{-(j \wedge k)-N} \ell^{\prime}, 2^{-(j \wedge 2 k)-N} \ell_{m}\right)\right|^{2} \chi_{I} \chi_{J}\right\}^{1 / 2}\right\|_{L^{p}} \\
& =C \|\left\{\sum_{j, k} \sum_{\left(\ell^{\prime}, \ell_{m}\right)} \mid \sum_{j^{\prime}, k^{\prime}\left(\ell^{\prime \prime}, \ell_{m}^{\prime}\right)} 2^{-(m-1)\left(j^{\prime} \wedge k^{\prime}\right)} 2^{-\left(j^{\prime} \wedge 2 k^{\prime}\right)}\right. \\
& \times\left(\phi_{j^{\prime}, k^{\prime}} * h\right)\left(2^{-\left(j^{\prime} \wedge k^{\prime}\right)-N} \ell^{\prime \prime}, 2^{-\left(j^{\prime} \wedge 2 k^{\prime}\right)-N} \ell_{m}^{\prime}\right) \\
& \times\left.\left(\mathcal{K} * \phi_{j, k} * \phi_{j^{\prime}, k^{\prime}}\right)\left(2^{-(j \wedge k)-N} \ell^{\prime}-2^{-\left(j^{\prime} \wedge k^{\prime}\right)-N} \ell^{\prime \prime}, 2^{-(j \wedge 2 k)-N} \ell_{m}-2^{-\left(j^{\prime} \wedge 2 k^{\prime}\right)-N} \ell_{m}^{\prime}\right)\right|^{2} \\
& \left.\quad \times \chi_{I} \chi_{J}\right\}^{1 / 2} \|_{L^{p}},
\end{aligned}
$$

where $\phi_{j, k}, \phi_{j^{\prime}, k^{\prime}}, h$ and $N$ are the same as in Theorem 4.1. 
We claim that for any given $M>0$,

$$
\left|\mathcal{K}_{1} * \phi_{k}^{(1)}\left(x^{\prime}, x_{m}\right)\right| \leq C \frac{2^{k m}}{\left(1+2^{k}\left|x^{\prime}\right|\right)^{M+m-1}\left(1+2^{k}\left|x_{m}\right|\right)^{M+1}},
$$

and

$$
\left|\mathcal{K}_{2} * \phi_{k}^{(2)}\left(x^{\prime}, x_{m}\right)\right| \leq C \frac{2^{k(m+1)}}{\left(1+2^{k}\left|x^{\prime}\right|\right)^{M+m+1}\left(1+2^{2 k}\left|x_{m}\right|\right)^{M+1}} .
$$

We only prove (4.12) here, since the proof of (4.11) is similar. We consider the following two cases:

Case 1. $|x|_{h} \leq 22^{-k}$.

In this case, $2^{k}\left|x^{\prime}\right| \leq 2$ and $2^{2 k}\left|x_{m}\right| \leq 4$, which imply that

$$
1+2^{k}\left|x^{\prime}\right| \sim 1 \text { and } 1+2^{2 k}\left|x_{m}\right| \sim 1 \text {. }
$$

By the fact supp $\phi_{k}^{(2)} \subset\left\{x:|x|_{h} \leq 2^{-k}\right\}$ and the cancellation condition in (4.4), $\mathcal{K}_{2} * \phi_{k}^{(2)}(x)$ is bounded by

$$
\begin{aligned}
\mid \mathcal{K}_{2} * & \phi_{k}^{(2)}(x)|=| \lim _{\varepsilon \rightarrow 0} \int_{\varepsilon \leq|x-y|_{h} \leq 102^{-k}} \mathcal{K}_{2}(x-y) \phi_{k}^{(2)}(y) d y \mid \\
& =\left|\lim _{\varepsilon \rightarrow 0} \int_{\varepsilon \leq|x-y|_{h} \leq 32^{-k}} \mathcal{K}_{2}(x-y)\left[\phi_{k}^{(2)}(y)-\phi_{k}^{(2)}(x)\right] d y\right| \\
& \leq C 2^{k(m+1)} \int_{\left|x^{\prime}-y^{\prime}\right| \leq 3}\left(\left|x^{\prime}-y^{\prime}\right|\right)^{-(m-1)+1} d y^{\prime} \int_{\left|x_{m}-y_{m}\right| \leq 9}\left|x_{m}-y_{m}\right|^{-2+2} d y_{m} \\
& \leq C 2^{k(m+1)} \leq C \frac{2^{k(m+1)}}{\left(1+2^{k}\left|x^{\prime}\right|\right)^{M+m-1}\left(1+2^{2 k}\left|x_{m}\right|\right)^{M+1}} .
\end{aligned}
$$

Case 2. $|x|_{h}>22^{-k}$.

In this case, $2^{k}\left|x^{\prime}\right|>2$ or $2^{2 k}\left|x_{m}\right|>4$, which imply that

$$
1+2^{k}\left|x^{\prime}\right| \sim 2^{k}\left|x^{\prime}\right| \text { or } 1+2^{2 k}\left|x_{m}\right| \sim 2^{2 k}\left|x_{m}\right| .
$$

By the cancellation condition of $\phi^{(2)}$ with order $4 M$ in (4.4) and the size condition of $\mathcal{K}_{2}$ in (1.3),

$$
\begin{aligned}
& \left|\mathcal{K}_{2} * \phi_{k}^{(2)}(x)\right| \\
& =\left|\int_{|y|_{h} \leq 2^{-k}}\left[\mathcal{K}_{2}(x-y)-\sum_{|\alpha|=\left|\alpha_{1}\right|+\left|2 \alpha_{2}\right| \leq 4 M} \frac{1}{\alpha !} D_{x^{\prime}}^{\alpha_{1}} D_{x_{m}}^{\alpha_{2}} \mathcal{K}_{2}\left(x^{\prime}, x_{m}\right) y^{\alpha}\right] \phi_{k}^{(2)}(y) d y\right| \\
& \leq C \int_{|y|_{h} \leq 2^{-k}} \frac{\left(|y|_{h}\right)^{4 M+1}}{\left(|x|_{h}\right)^{m+1+4 M+1}\left|\phi_{k}^{(2)}(y)\right| d y} \\
& \leq C \frac{2^{k(m+1)}}{\left(1+2^{k}\left|x^{\prime}\right|\right)^{M+m-1}\left(1+2^{2 k}\left|x_{m}\right|\right)^{M+1}} .
\end{aligned}
$$

The claim follows. 
By the classical orthogonality argument, for any fixed $L$ and $M$,

$$
\left|\phi_{j}^{(1)} * \phi_{j^{\prime}}^{(1)}\left(x^{\prime}, x_{m}\right)\right| \leq C \frac{2^{-\left|j-j^{\prime}\right| L} 2^{m\left(j \wedge j^{\prime}\right)}}{\left(1+2^{\left(j \wedge j^{\prime}\right)}\left|x^{\prime}\right|\right)^{(M+m-1)}\left(1+2^{\left(j \wedge j^{\prime}\right)}\left|x_{m}\right|\right)^{(M+1)}},
$$

and

$$
\left|\phi_{k}^{(2)} * \phi_{k^{\prime}}^{(2)}\left(x^{\prime}, x_{m}\right)\right| \leq C \frac{2^{-\left|k-k^{\prime}\right| L} 2^{\left(k \wedge k^{\prime}\right)(m+1)}}{\left(1+2^{\left(k \wedge k^{\prime}\right)}\left|x^{\prime}\right|\right)^{(M+m-1)}\left(1+2^{2\left(k \wedge k^{\prime}\right)}\left|x_{m}\right|\right)^{(M+1)}} .
$$

The estimates (4.11)-(4.14) yield that

$$
\begin{aligned}
& \left|\mathcal{K} * \phi_{j, k} * \phi_{j^{\prime}, k^{\prime}}\left(x^{\prime}, x_{m}\right)\right|=\left|\left[\mathcal{K}_{1} * \phi_{j}^{(1)} * \phi_{j^{\prime}}^{(1)}\right] *\left[\mathcal{K}_{2} * \psi_{k}^{(2)} * \psi_{k^{\prime}}^{(2)}\right]\left(x^{\prime}, x_{m}\right)\right| \\
& \quad \leq C \frac{2^{-\left|j-j^{\prime}\right| L} 2^{-\left|k-k^{\prime}\right| L} 2^{\left(j \wedge j^{\prime} \wedge k \wedge k^{\prime}\right)(m-1)} 2^{j \wedge j^{\prime} \wedge 2 k \wedge 2 k^{\prime}}}{\left(1+2^{j \wedge j^{\prime} \wedge k \wedge k^{\prime}}\left|x^{\prime}\right|\right)^{(M+m-1)}\left(1+2^{j \wedge j^{\prime} \wedge 2 k \wedge 2 k^{\prime}}\left|x_{m}\right|\right)^{(M+1)}} .
\end{aligned}
$$

Using the estimates in (4.15) and applying the same proof as in Theorem 1.6 yield that for $f \in L^{2} \cap H_{\mathrm{com}}^{p}$ and $0<\delta<p \leq 1$,

$$
\begin{aligned}
\|T(f)\|_{H_{\mathrm{com}}^{p}} & \leq C \|\left\{\sum _ { j ^ { \prime } , k ^ { \prime } } \left\{\mathcal { M } _ { s } \left[\left(\sum_{\left(\ell^{\prime \prime}, \ell_{m}^{\prime}\right)}\left|\left(\phi_{j^{\prime}, k^{\prime}} * h\right)\left(2^{-\left(j^{\prime} \wedge k^{\prime}\right)-N} \ell^{\prime \prime}, 2^{-\left(j^{\prime} \wedge 2 k^{\prime}\right)-N} \ell_{m}^{\prime}\right)\right|\right.\right.\right.\right. \\
& \leq C\|h\|_{H_{\mathrm{com}}^{p}} \leq C\|f\|_{H_{\mathrm{com}}^{p}} .
\end{aligned}
$$

Since $L^{2} \cap H_{\text {com }}^{p}$ is dense in $H_{\text {com }}^{p}$, this concludes the proof of Theorem 1.7.

\section{Proofs of Theorems 1.8 and 1.9}

In this section we prove Theorem 1.8 using Theorem 4.1. Theorem 1.9 then follows directly from Theorem 1.8.

Proof of Theorem 1.8. For any $f \in L^{2}\left(\mathbb{R}^{m}\right) \cap H_{\text {com }}^{p}\left(\mathbb{R}^{m}\right)$, set

$$
\Omega_{i}=\left\{\left(x^{\prime}, x_{m}\right) \in \mathbb{R}^{m-1} \times \mathbb{R}: \widetilde{\mathcal{G}}_{\phi}^{d}(f)\left(x^{\prime}, x_{m}\right)>2^{i}\right\},
$$

where

$\widetilde{\mathcal{G}}_{\phi}^{d}(f)=\left\{\sum_{j, k \in \mathbb{Z}\left(\ell^{\prime}, \ell_{m}\right) \in \mathbb{Z}^{m-1} \times \mathbb{Z}}\left|\left(\phi_{j, k} * h\right)\left(2^{-(j \wedge k)-N} \ell^{\prime}, 2^{-(j \wedge 2 k)-N} \ell_{m}\right)\right|^{2} \chi_{I} \chi_{J}\right\}^{1 / 2}$.

Here $\phi_{j, k}$ and $h$ are given by Theorem 4.1. Write

$$
\mathcal{B}_{i}=\left\{(j, k, I, J):\left|(I \times J) \cap \Omega_{i}\right|>\frac{1}{2}|I \times J|, \quad\left|(I \times J) \cap \Omega_{i+1}\right| \leq \frac{1}{2}|I \times J|\right\},
$$

where the $I$ are dyadic cubes in $\mathbb{R}^{m-1}$ and the $J$ are dyadic intervals in $\mathbb{R}$ with side lengths $\ell(I)=2^{-(j \wedge k)-N}$ and $\ell(J)=2^{-(j \wedge 2 k)-N}$, and the lower left corners of $I$ and the left endpoints of $J$ are $2^{-(j \wedge k)-N} \ell^{\prime}$ and $2^{-(j \wedge 2 k)-N} \ell_{m}$, respectively. 
Using Theorem 4.1, we write

$$
\begin{aligned}
f\left(x^{\prime}, x_{m}\right)=\sum_{i} \sum_{(j, k, I, J) \in \mathcal{B}_{i}}|I||J| \phi_{j, k}( & \left.x^{\prime}-2^{-(j \wedge k)-N} \ell^{\prime}, x_{m}-2^{-(j \wedge 2 k)-N} \ell_{m}\right) \\
& \times\left(\phi_{j, k} * h\right)\left(2^{-(j \wedge k)-N} \ell^{\prime}, 2^{-(j \wedge 2 k)-N} \ell_{m}\right),
\end{aligned}
$$

where the series converges in the $L^{2}$ norm. We claim that

$$
\begin{aligned}
\left\|\sum_{(j, k, I, J) \in \mathcal{B}_{i}}|I \| J| \phi_{j, k}(\cdot\right. & \left.-2^{-(j \wedge k)-N} \ell^{\prime}, \cdot-2^{-(j \wedge 2 k)-N} \ell_{m}\right) \\
& \times\left(\phi_{j, k} * h\right)\left(2^{-(j \wedge k)-N} \ell^{\prime}, 2^{-(j \wedge 2 k)-N} \ell_{m}\right) \|_{L^{p}}^{p} \leq C 2^{i p}\left|\Omega_{i}\right|,
\end{aligned}
$$

which together with $0<p \leq 1$ yields

$$
\|f\|_{L^{p}}^{p} \leq C \sum_{i} 2^{i p}\left|\Omega_{i}\right| \leq C\left\|\widetilde{\mathcal{G}}_{\phi}^{d}(f)\right\|_{L^{p}}^{p} \leq C\|h\|_{H_{\mathrm{com}}^{p}}^{p} \leq C\|f\|_{H_{\mathrm{com}}^{p}}^{p} .
$$

Now we show the claim. Note that the functions $\phi^{(1)}$ and $\phi^{(2)}$ are supported in unit balls. Hence if $(j, k, I, J) \in \mathcal{B}_{i}$, then the $\phi_{j, k}$ are supported in

$$
\widetilde{\Omega}_{i}=\left\{\left(x^{\prime}, x_{m}\right): M_{s}\left(\chi_{\Omega_{i}}\right)\left(x^{\prime}, x_{m}\right)>\frac{1}{100^{m}}\right\} .
$$

For the sake of convenience, we denote $x_{I}=2^{-(j \wedge k)-N} \ell^{\prime}$ and $x_{J}=2^{-(j \wedge 2 k)-N} \ell_{m}$. Since $\left|\widetilde{\Omega}_{i}\right| \leq C\left|\Omega_{i}\right|$, by Hölder's inequality we obtain

$$
\begin{aligned}
& \left\|\sum_{(j, k, I, J) \in \mathcal{B}_{i}}|I||J| \phi_{j, k}\left(\cdot-x_{I}, \cdot-x_{J}\right)\left(\phi_{j, k} * h\right)\left(x_{I}, x_{J}\right)\right\|_{L^{p}}^{p} \\
& \quad \leq\left|\Omega_{i}\right|^{1-p / 2}\left\|\sum_{(j, k, I, J) \in \mathcal{B}_{i}}|I||J| \phi_{j, k}\left(\cdot-x_{I}, \cdot-x_{J}\right)\left(\phi_{j, k} * h\right)\left(x_{I}, x_{J}\right)\right\|_{2}^{p} .
\end{aligned}
$$

By the duality argument, we estimate the $L^{2}$ norm of

$$
\sum_{(j, k, I, J) \in \mathcal{B}_{i}}|I||J| \phi_{j, k}\left(\cdot-x_{I}, \cdot-x_{J}\right)\left(\phi_{j, k} * h\right)\left(x_{I}, x_{J}\right)
$$

as follows: for all $g \in L^{2}$ with $\|g\|_{2} \leq 1$,

$$
\begin{aligned}
\left|<\sum_{(j, k, I, J) \in \mathcal{B}_{i}}\right| I|| J \mid \phi_{j, k}( & \left.-x_{I}, \cdot-x_{J}\right)\left(\phi_{j, k} * h\right)\left(x_{I}, x_{J}\right), g>\mid \\
\leq C & \left(\sum_{(j, k, I, J) \in \mathcal{B}_{i}}|I||J|\left|\left(\phi_{j, k} * h\right)\left(x_{I}, x_{J}\right)\right|^{2}\right)^{1 / 2} \\
& \times\left(\sum_{(j, k, I, J) \in \mathcal{B}_{i}}|I||J|\left|\left(\phi_{j, k} * g\right)\left(x_{I}, x_{J}\right)\right|^{2}\right)^{1 / 2} .
\end{aligned}
$$


At the same time,

$$
\begin{aligned}
& \sum_{(j, k, I, J) \in \mathcal{B}_{i}}|I||J|\left|\left(\phi_{j, k} * g\right)\left(x_{I}, x_{J}\right)\right|^{2} \\
& \left.\quad=\left.\int_{\mathbb{R}^{m-1} \times \mathbb{R}}\left\{\sum_{(j, k, I, J) \in \mathcal{B}_{i}} \mid\left(\phi_{j, k} * g\right)\left(x_{I}, x_{J}\right)\right)\right|^{2} \chi_{I}\left(x^{\prime}\right) \chi_{J}\left(x_{m}\right)\right\}^{(1 / 2) \cdot 2} d x^{\prime} d x_{m} \\
& \leq \int_{\mathbb{R}^{m-1} \times \mathbb{R}} \widetilde{\mathcal{G}}_{\phi}^{d}(g)\left(x^{\prime}, x_{m}\right)^{2} d x^{\prime} d x_{m} \leq\|g\|_{L^{2}}^{2} .
\end{aligned}
$$

In addition,

$$
\begin{aligned}
C 2^{2 i}\left|\Omega_{i}\right| & \geq \int_{\widetilde{\Omega}_{i} \backslash \Omega_{i+1}}\left[\widetilde{\mathcal{G}}_{\phi}^{d}(f)(x, y)\right]^{2} d x^{\prime} d x_{m} \\
& \geq \sum_{(j, k, I, J) \in \mathcal{B}_{i}}\left|\left(\phi_{j, k} * h\right)\left(x_{I}, x_{J}\right)\right|^{2}\left|(I \times J) \cap \widetilde{\Omega}_{i} \backslash \Omega_{i+1}\right| \\
& \geq \frac{1}{2} \sum_{(j, k, I, J) \in \mathcal{B}_{i}}|I||J|\left|\left(\phi_{j, k} * h\right)\left(x_{I}, x_{J}\right)\right|^{2},
\end{aligned}
$$

where in the last inequality we use the fact that $\left|(I \times J) \cap \widetilde{\Omega_{i}} \backslash \Omega_{i+1}\right|>\frac{1}{2}|I \times J|$ when $(j, k, I, J) \in \mathcal{B}_{i}$. This completes the proof of Theorem 1.8.

Proof of Theorem 1.9. Suppose $f \in H_{\mathrm{com}}^{p} \cap L^{2}$. By Theorem 1.7, $T$ is bounded on $H_{\mathrm{com}}^{p}$, which together with the fact that $T$ is also bounded on $L^{2}$ yields that $T(f) \in H_{\mathrm{com}}^{p} \cap L^{2}$, so applying first Theorem 1.8 and then Theorem 1.7 we obtain

$$
\|T(f)\|_{L^{p}} \leq C\|T(f)\|_{H_{\mathrm{com}}^{p}} \leq C\|f\|_{H_{\mathrm{com}}^{p}} \text { for any } f \in L^{2} \cap H_{\mathrm{com}}^{p} .
$$

Since $H_{\mathrm{com}}^{p} \cap L^{2}$ is dense in $H_{\mathrm{com}}^{p}$, the composition operator $T$ extends to a bounded operator from $H_{\text {com }}^{p}$ to $L^{p}$.

\section{Proofs of Theorems 1.10 and 1.11}

We now prove the Calderón-Zygmund decomposition and the interpolation theorem on $H_{\text {com }}^{p}\left(\mathbb{R}^{m}\right)$.

Proof of Theorem 1.10. We first assume $f \in L^{2} \cap H_{\text {com }}^{p}$. Let $\alpha>0$ and $\Omega_{\ell}=\{x \in$ $\left.\mathbb{R}^{m}: \widetilde{\mathcal{G}}_{\phi}^{d}(f)(x)>\alpha 2^{\ell}\right\}$, where $\widetilde{\mathcal{G}}_{\phi}^{d}(f)$ is defined as in the the proof of Theorem 1.8. Let

$$
\mathcal{R}_{0}=\left\{I \times J:\left|(I \times J) \cap \Omega_{0}\right|<\frac{1}{2}|I \times J|\right\},
$$

and for $\ell \geq 1$,

$$
\mathcal{R}_{\ell}=\left\{I \times J:\left|(I \times J) \cap \Omega_{\ell-1}\right| \geq \frac{1}{2}|I \times J|,\left|(I \times J) \cap \Omega_{\ell}\right|<\frac{1}{2}|I \times J|\right\},
$$

where the $I$ are dyadic cubes in $\mathbb{R}^{m-1}$ and the $J$ are dyadic intervals in $\mathbb{R}$ with side lengths $\ell(I)=2^{-(j \wedge k)-N}$ and $\ell(J)=2^{-(j \wedge 2 k)-N}$, and the lower left corners of $I$ and the left endpoints of $J$ are $2^{-(j \wedge k)-N} \ell^{\prime}$ and $2^{-(j \wedge 2 k)-N} \ell_{m}$, respectively. 
By the discrete Calderón-type identity of Theorem 4.1,

$$
\begin{aligned}
f\left(x^{\prime}, x_{m}\right)= & \sum_{j, k} \sum_{I, J}|I||J| \phi_{j, k}\left(x^{\prime}-x_{I}, x_{m}-y_{J}\right) \phi_{j, k} * h\left(x_{I}, y_{J}\right) \\
= & \sum_{j, k} \sum_{\ell \geq 1} \sum_{I \times J \in \mathcal{R}_{\ell}}|I||J| \phi_{j, k}\left(x^{\prime}-x_{I}, x_{m}-y_{J}\right) \phi_{j, k} * h\left(x_{I}, y_{J}\right) \\
& +\sum_{j, k} \sum_{I \times J \in \mathcal{R}_{0}}|I||J| \phi_{j, k}\left(x^{\prime}-x_{I}, x_{m}-y_{J}\right) \phi_{j, k} * h\left(x_{I}, y_{J}\right) \\
= & b\left(x^{\prime}, x_{m}\right)+g\left(x^{\prime}, x_{m}\right),
\end{aligned}
$$

where $x_{I}=2^{-(j \wedge k)-N} \ell^{\prime}$ and $y_{J}=2^{-(j \wedge 2 k)-N} \ell_{m}$.

When $p_{1}>1$, using a duality argument as in the proof of Theorem 1.8, it is easy to show that

$$
\|g\|_{p_{1}} \leq C\left\|\left\{\sum_{j, k} \sum_{I \times J \in \mathcal{R}_{0}}\left|\phi_{j, k} * h\left(x_{I}, y_{J}\right)\right|^{2} \chi_{I} \chi_{J}\right\}^{1 / 2}\right\|_{p_{1}}
$$

Next, we estimate $\|g\|_{H_{\mathrm{com}}^{p_{1}}}$ when $0<p_{1} \leq 1$. Clearly, the duality argument will not work here. Nevertheless, we can estimate the $H_{\text {com }}^{p_{1}}$ norm directly by using discrete Calderón's identity in Theorem 1.3. To this end, we note that

$$
\|g\|_{H_{\mathrm{com}}^{p_{1}}} \leq\left\|\left\{\sum_{j^{\prime}, k^{\prime}} \sum_{I^{\prime}, J^{\prime}}\left|\left(\psi_{j^{\prime} k^{\prime}} * g\right)\left(x_{I^{\prime}}, y_{J^{\prime}}\right)\right|^{2} \chi_{I^{\prime}}(x) \chi_{J^{\prime}}(y)\right\}^{1 / 2}\right\|_{L^{p_{1}}},
$$

where the $I^{\prime}$ are dyadic cubes in $\mathbb{R}^{m-1}$ and the $J^{\prime}$ are dyadic intervals in $\mathbb{R}$ with side lengths $\ell\left(I^{\prime}\right)=2^{-\left(j^{\prime} \wedge k^{\prime}\right)}$ and $\ell\left(J^{\prime}\right)=2^{-\left(j^{\prime} \wedge 2 k^{\prime}\right)}$, and the lower left corners of $I^{\prime}$ and the left endpoints of $J^{\prime}$ are $2^{-\left(j^{\prime} \wedge k^{\prime}\right)} \ell^{\prime \prime}$ and $2^{-\left(j^{\prime} \wedge 2 k^{\prime}\right)} \ell_{m}^{\prime}$, respectively.

Since

$\left(\psi_{j^{\prime}, k^{\prime}} * g\right)\left(x_{I^{\prime}}, y_{J^{\prime}}\right)=\sum_{j, k} \sum_{I \times J \in \mathcal{R}_{0}}|I||J|\left(\psi_{j^{\prime} k^{\prime}} * \phi_{j, k}\right)\left(x_{I^{\prime}}-x_{I}, y_{J^{\prime}}-y_{J}\right) \phi_{j, k} * h\left(x_{I}, y_{J}\right)$,

we have, by following the proof of Theorem 1.6, that

$$
\begin{gathered}
\|\left\{\sum_{j^{\prime}, k^{\prime}} \sum_{I^{\prime}, J^{\prime}} \mid\left(\psi_{j^{\prime} k^{\prime}} * g\right)\right. \\
\left.\left.\quad\left(x_{I^{\prime}}, y_{J^{\prime}}\right)\right|^{2} \chi_{I^{\prime}}(x) \chi_{J^{\prime}}(y)\right\}^{1 / 2} \|_{p_{1}} \\
\leq C\left\|\left\{\sum_{j, k} \sum_{I \times J \in \mathcal{R}_{0}}\left|\phi_{j, k} * h\left(x_{I}, y_{J}\right)\right|^{2} \chi_{I} \chi_{J}\right\}^{1 / 2}\right\|_{p_{1}}
\end{gathered}
$$

This shows that, for all $0<p_{1}<\infty$,

$$
\|g\|_{H_{\mathrm{com}}^{p_{1}}} \leq C\left\|\left\{\sum_{j, k} \sum_{I \times J \in \mathcal{R}_{0}}\left|\phi_{j, k} * h\left(x_{I}, y_{J}\right)\right|^{2} \chi_{I} \chi_{J}\right\}^{1 / 2}\right\|_{p_{1}} .
$$




\section{Claim 1.}

$$
\begin{aligned}
\int_{\widetilde{\mathcal{G}}^{d}(f)\left(x^{\prime}, x_{m}\right) \leq \alpha}\left(\widetilde{\mathcal{G}}^{d}(f)\right)^{p_{1}}\left(x^{\prime}, x_{m}\right) d x^{\prime} d x_{m} \\
\geq C\left\|\left\{\sum_{j, k} \sum_{I \times J \in \mathcal{R}_{0}}\left|\phi_{j, k} * h\left(x_{I}, y_{J}\right)\right|^{2} \chi_{I} \chi_{J}\right\}^{1 / 2}\right\|_{p_{1}}^{p_{1}}
\end{aligned}
$$

This claim implies

$$
\begin{aligned}
\|g\|_{H_{\mathrm{com}}}^{p_{1}} & \leq C \int_{\widetilde{\mathcal{G}}^{d}(f)\left(x^{\prime}, x_{m}\right) \leq \alpha}\left(\widetilde{\mathcal{G}}^{d}(f)\right)^{p_{1}}\left(x^{\prime}, x_{m}\right) d x^{\prime} d x_{m} \\
& \leq C \alpha^{p_{1}-p} \int_{\widetilde{\mathcal{G}}^{d}(f)\left(x^{\prime}, x_{m}\right) \leq \alpha}\left(\widetilde{\mathcal{G}}^{d}(f)\right)^{p}\left(x^{\prime}, x_{m}\right) d x^{\prime} d x_{m} \\
& \leq C \alpha^{p_{1}-p}\|f\|_{H_{\mathrm{com}}^{p}}^{p} .
\end{aligned}
$$

To show Claim 1, we choose $0<q<p_{1}$ and note that

$$
\begin{aligned}
& \int_{\widetilde{\mathcal{G}}^{d}(f)\left(x^{\prime}, x_{m}\right) \leq \alpha}\left(\widetilde{\mathcal{G}}^{d}(f)\right)^{p_{1}}\left(x^{\prime}, x_{m}\right) d x^{\prime} d x_{m} \\
& =\int_{\widetilde{\mathcal{G}}^{d}(f)\left(x^{\prime}, x_{m}\right) \leq \alpha}\left\{\sum_{j, k} \sum_{I, J}\left|\phi_{j, k} * h\left(x_{I}, y_{J}\right)\right|^{2} \chi_{I}\left(x^{\prime}\right) \chi_{J}\left(x_{m}\right)\right\}^{p_{1} / 2} d x^{\prime} d x_{m} \\
& \geq C \int_{\Omega_{0}^{c}}\left\{\sum_{j, k} \sum_{R=I \times J \in \mathcal{R}_{0}}\left|\phi_{j, k} * h\left(x_{I}, y_{J}\right)\right|^{2} \chi_{I} \chi_{J}\right\}^{p_{1} / 2} d x^{\prime} d x_{m} \\
& =C \int_{\mathbb{R}^{m-1} \times \mathbb{R}}\left\{\sum_{j, k} \sum_{R \in \mathcal{R}_{0}}\left|\phi_{j, k} * h\left(x_{I}, y_{J}\right)\right|^{2} \chi_{R \cap \Omega_{0}^{c}}\left(x^{\prime}, x_{m}\right)\right\}^{p_{1} / 2} d x^{\prime} d x_{m} \\
& \geq C \int_{\mathbb{R}^{m-1} \times \mathbb{R}}\left\{\left\{\sum_{j, k} \sum_{R \in \mathcal{R}_{0}}\left(M_{s}\left(\left|\phi_{j, k} * h\left(x_{I}, y_{J}\right)\right|^{q} \chi_{R \cap \Omega_{0}^{c}}\right)\left(x^{\prime}, x_{m}\right)\right)^{2 / q}\right\}^{q / 2}\right\}^{p_{1} / q} \\
& \times d x^{\prime} d x_{m} \\
& \geq C \int_{\mathbb{R}^{m-1} \times \mathbb{R}}\left\{\sum_{j, k} \sum_{R \in \mathcal{R}_{0}}\left|\phi_{j, k} * h\left(x_{I}, y_{J}\right)\right|^{2} \chi_{R}\left(x^{\prime}, x_{m}\right)\right\}^{p_{1} / 2} d x^{\prime} d x_{m}
\end{aligned}
$$

where in the last inequality we have used the fact that $\left|\Omega_{0}^{c} \cap R\right| \geq \frac{1}{2}|R|$ for $R=$ $I \times J \in \mathcal{R}_{0}$, and thus

$$
\chi_{I}\left(x^{\prime}\right) \chi_{J}\left(x_{m}\right) \leq 2^{1 / q} M_{s}\left(\chi_{R \cap \Omega_{0}^{c}}\right)^{1 / q}\left(x^{\prime}, x_{m}\right),
$$

and in the penultimate inequality we have used the vector-valued Fefferman-Stein inequality for strong maximal functions

$$
\left\|\left(\sum_{k=1}^{\infty}\left(M_{s}\left(f_{k}\right)\right)^{r}\right)^{1 / r}\right\|_{p} \leq C\left\|\left(\sum_{k=1}^{\infty}\left|f_{k}\right|^{r}\right)^{1 / r}\right\|_{p}
$$

with the exponents $r=2 / q>1$ and $p=p_{1} / q>1$. The claim follows. 
We now recall $\widetilde{\Omega}_{\ell}=\left\{\left(x^{\prime}, x_{m}\right) \in \mathbb{R}^{m-1} \times \mathbb{R}: M_{s}\left(\chi_{\Omega_{\ell}}\right)>\frac{1}{2}\right\}$.

Claim 2. For any $0<p_{2} \leq 1$ and $\ell \geq 1$,

$$
\left\|\sum_{j, k} \sum_{I \times J \in \mathcal{R}_{\ell}}|I||J| \widetilde{\phi}_{j, k}\left(x^{\prime}-x_{I}, x_{m}-y_{J}\right) \phi_{j, k} * h\left(x_{I}, y_{J}\right)\right\|_{H_{\mathrm{com}}^{p_{2}}}^{p_{2}} \leq C\left(2^{\ell} \alpha\right)^{p_{2}} \widetilde{\Omega_{\ell-1} \mid} .
$$

Claim 2 implies

$$
\begin{aligned}
\|b\|_{H_{\mathrm{com}}^{p_{2}}}^{p_{2}} & \leq \sum_{\ell \geq 1}\left(2^{\ell} \alpha\right)^{p_{2}} \mid \widetilde{\Omega_{\ell-1} \mid} \\
& \leq C \sum_{\ell \geq 1}\left(2^{\ell} \alpha\right)^{p_{2}}\left|\Omega_{\ell-1}\right| \leq C \int_{\widetilde{\mathcal{G}}^{d}(f)(x, y)>\alpha}\left(\widetilde{\mathcal{G}}^{d}\right)^{p_{2}}(f)\left(x^{\prime}, x_{m}\right) d x^{\prime} d x_{m} \\
& \leq C \alpha^{p_{2}-p} \int_{\widetilde{\mathcal{G}}^{d}(f)(x, y)>\alpha}\left(\widetilde{\mathcal{G}}^{d}\right)^{p}(f)\left(x^{\prime}, x_{m}\right) d x^{\prime} d x_{m} \leq C \alpha^{p_{2}-p}\|f\|_{H_{\mathrm{com}}^{p}}^{p} .
\end{aligned}
$$

To show Claim 2, again we have

$$
\begin{gathered}
\left\|\sum_{j, k} \sum_{I \times J \in \mathcal{R}_{\ell}}|I||J| \phi_{j, k}\left(x^{\prime}-x_{I}, x_{m}-y_{J}\right) \phi_{j, k} * h\left(x_{I}, y_{J}\right)\right\|_{H_{\mathrm{com}}^{p_{2}}} \\
\leq C \|\left\{\sum_{j^{\prime} k^{\prime}} \sum_{I^{\prime}, J^{\prime}}\left|\sum_{j, k} \sum_{I \times J \in \mathcal{R}_{\ell}}\right| I|| J \mid\left(\psi_{j^{\prime} k^{\prime}} * \phi_{j, k}\right)\left(x_{I^{\prime}}-x_{I}, y_{J^{\prime}}-y_{J}\right) \phi_{j, k}\right. \\
\left.\left.\quad * h\left(x_{I}, y_{J}\right)\right|^{2}\right\}^{1 / 2} \|_{L^{p_{2}}} \\
\leq C\left\|\left\{\sum_{j, k} \sum_{I \times J \in \mathcal{R}_{\ell}}\left|\phi_{j, k} * h\left(x_{I}, y_{J}\right)\right|^{2} \chi_{I} \chi_{J}\right\}^{1 / 2}\right\|_{L^{p_{2}}}
\end{gathered}
$$

where we can use an argument similar to that in the proof of Theorem 1.8 to prove the last inequality.

However, as in the proof of the first claim, choosing $0<q<2$ and $q<p_{2}$ implies that

$$
\begin{aligned}
\left(2^{\ell} \alpha\right)^{p_{2}}\left|\widetilde{\Omega}_{\ell-1}\right| \geq \int_{\widetilde{\Omega}_{\ell-1} \backslash \Omega_{\ell}} \widetilde{\mathcal{G}}^{d}(f)^{p_{2}}\left(x^{\prime}, x_{m}\right) d x^{\prime} d x_{m} \\
\quad=\int_{\widetilde{\Omega}_{\ell-1} \backslash \Omega_{\ell}}\left\{\sum_{j, k} \sum_{I, J}\left|\phi_{j, k} * h\left(x_{I}, y_{J}\right)\right|^{2} \chi_{I}\left(x^{\prime}\right) \chi_{J}\left(x_{m}\right)\right\}^{p_{2} / 2} d x^{\prime} d x_{m} \\
=\int_{\mathbb{R}^{m-1} \times \mathbb{R}}\left\{\sum_{j, k} \sum_{I, J}\left|\phi_{j, k} * h\left(x_{I}, y_{J}\right)\right|^{2} \chi_{\left.(I \times J) \cap \widetilde{\Omega}_{\ell-1} \backslash \Omega_{\ell}\right)}\left(x^{\prime}, x_{m}\right)\right\}^{p_{2} / 2} d x^{\prime} d x_{m} \\
\geq C \int_{\mathbb{R}^{m-1} \times \mathbb{R}}\left\{\left\{\sum _ { j , k } \sum _ { I , J } \left(M _ { s } \left(\left|\phi_{j, k} * h\left(x_{I}, y_{J}\right)\right|^{q}\right.\right.\right.\right. \\
\geq C \int_{\mathbb{R}^{m-1} \times \mathbb{R}}\left\{\sum_{I \times J \in \mathcal{R}_{\ell}}\left|\phi_{j, k} * h\left(x_{I}, y_{J}\right)\right|^{2} \chi_{I}\left(x^{\prime}\right) \chi_{J}\left(x_{m}\right)\right\}^{p_{2} / 2} d x^{\prime} d x_{m} .
\end{aligned}
$$


In the above string of inequalities, we have used the fact that for $I \times J \in \mathcal{R}_{\ell}$ we have

$$
\left|(I \times J) \cap \Omega_{\ell-1}\right|>\frac{1}{2}|I \times J| \quad \text { and } \quad\left|(I \times J) \cap \Omega_{\ell}\right| \leq \frac{1}{2}|I \times J|,
$$

and consequently $I \times J \subset \widetilde{\Omega}_{\ell-1}$. Therefore $\left|(I \times J) \cap\left(\widetilde{\Omega}_{\ell-1} \backslash \Omega_{\ell}\right)\right|>\frac{1}{2}|I \times J|$ for $I \times J \in \mathcal{R}_{\ell}$. Thus

$$
\chi_{I}\left(x^{\prime}\right) \chi_{J}\left(x_{m}\right) \leq 2^{1 / q} M_{s}\left(\chi_{\left.(I \times J) \cap \widetilde{\Omega}_{\ell-1} \backslash \Omega_{\ell}\right)}\right)^{1 / q}\left(x^{\prime}, x_{m}\right) .
$$

This gives the proof of the second claim. Since $L^{2}\left(\mathbb{R}^{m}\right) \cap H_{\text {com }}^{p}$ is dense in $H_{\text {com }}^{p}$.

We are now ready to prove the interpolation theorem on Hardy spaces $H_{\text {com }}^{p}$ for all $0<p<\infty$.

Proof of Theorem 1.11. Suppose that $T$ is bounded from $H_{\mathrm{com}}^{p_{2}}$ to $L^{p_{2}}$ and from $H_{\mathrm{com}}^{p_{1}}$ to $L^{p_{1}}$. For any given $\lambda>0$ and $f \in H_{\mathrm{com}}^{p}$, we have, by the CalderónZygmund decomposition,

$$
f(x)=g(x)+b(x)
$$

with

$$
\|g\|_{H_{\mathrm{com}}^{p_{1}}}^{p_{1}} \leq C \lambda^{p_{1}-p}\|f\|_{H_{\mathrm{com}}^{p}}^{p} \quad \text { and } \quad\|b\|_{H_{\mathrm{com}}^{p_{2}}}^{p_{2}} \leq C \lambda^{p_{2}-p}\|f\|_{H_{\mathrm{com}}^{p}}^{p} .
$$

Moreover, we have proved the estimates

$$
\|g\|_{H_{\mathrm{com}}^{p_{1}}}^{p_{1}} \leq C \int_{\widetilde{\mathcal{G}}^{d}(f)\left(x^{\prime}, x_{m}\right) \leq \alpha} \widetilde{\mathcal{G}}^{d}(f)^{p_{1}}\left(x^{\prime}, x_{m}\right) d x^{\prime} d x_{m}
$$

and

$$
\|b\|_{H_{\mathrm{com}}^{p_{2}}}^{p_{2}} \leq C \int_{\widetilde{\mathcal{G}}^{d}(f)\left(x^{\prime}, x_{m}\right)>\alpha} \widetilde{\mathcal{G}}^{d}(f)^{p_{2}}\left(x^{\prime}, x_{m}\right) d x^{\prime} d x_{m},
$$

which imply that

$$
\begin{aligned}
\|T f\|_{p}^{p}= & p \int_{0}^{\infty} \alpha^{p-1}\left|\left\{\left(x^{\prime}, x_{m}\right):\left|T f\left(x^{\prime}, x_{m}\right)\right|>\lambda\right\}\right| d \alpha \\
\leq & p \int_{0}^{\infty} \alpha^{p-1}\left|\left\{\left(x^{\prime}, x_{m}\right):\left|T g\left(x^{\prime}, x_{m}\right)\right|>\lambda / 2\right\}\right| d \alpha \\
& \quad+p \int_{0}^{\infty} \alpha^{p-1}\left|\left\{\left(x^{\prime}, x_{m}\right):\left|T b\left(x^{\prime}, x_{m}\right)\right|>\lambda / 2\right\}\right| d \alpha \\
\leq p & \int_{0}^{\infty} \alpha^{p-1} \int_{\widetilde{\mathcal{G}}^{d}(f)\left(x^{\prime}, x_{m}\right) \leq \alpha} \widetilde{\mathcal{G}}^{d}(f)^{p_{1}}\left(x^{\prime}, x_{m}\right) d x^{\prime} d x_{m} d \alpha \\
& \quad+p \int_{0}^{\infty} \alpha^{p-1} \int_{\widetilde{\mathcal{G}}^{d}(f)\left(x^{\prime}, x_{m}\right)>\alpha} \widetilde{\mathcal{G}}^{d}(f)^{p_{2}}\left(x^{\prime}, x_{m}\right) d x^{\prime} d x_{m} d \alpha \\
\leq C & \|f\|_{H_{\mathrm{com}}^{p}}^{p}
\end{aligned}
$$

Thus,

$$
\|T f\|_{p} \leq C\|f\|_{H_{\mathrm{com}}^{p}}
$$

for any $p_{2}<p<p_{1}$. Hence, $T$ is bounded from $H_{\mathrm{com}}^{p}$ to $L^{p}$. 
To prove the second assertion that $T$ is bounded on $H_{\mathrm{com}}^{p}$ for $p_{2}<p<p_{1}$, for any given $\lambda>0$ and $f \in H_{\text {com }}^{p}$, using again the Calderón-Zygmund decomposition gives

$$
\begin{aligned}
& \left|\left\{\left(x^{\prime}, x_{m}\right):\left|g(T f)\left(x^{\prime}, x_{m}\right)\right|>\alpha\right\}\right| \\
& \quad \leq\left|\left\{\left(x^{\prime}, x_{m}\right):\left|g(T g)\left(x^{\prime}, x_{m}\right)\right|>\alpha / 2\right\}\right|+\left|\left\{\left(x^{\prime}, x_{m}\right):\left|g(T b)\left(x^{\prime}, x_{m}\right)\right|>\alpha / 2\right\}\right| \\
& \leq C \alpha^{-p_{1}}\|T g\|_{H_{\mathrm{com}}^{p_{1}}}^{p_{1}}+C \alpha^{-p_{2}}\|T b\|_{H_{\mathrm{com}}^{p_{2}}}^{p_{2}} \leq C \alpha^{-p_{1}}\|g\|_{H_{\mathrm{com}}^{p_{1}}}^{p_{1}}+C \alpha^{-p_{2}}\|b\|_{H_{\mathrm{com}}}^{p_{2}} \\
& \quad \leq C \alpha^{-p_{1}} \int_{\widetilde{\mathcal{G}}^{d}(f)\left(x^{\prime}, x_{m}\right) \leq \alpha} \widetilde{\mathcal{G}}^{d}(f)^{p_{1}}\left(x^{\prime}, x_{m}\right) d x^{\prime} d x_{m} \\
& \quad+C \alpha^{-p_{2}} \int_{\widetilde{\mathcal{G}}^{d}(f)\left(x^{\prime}, x_{m}\right)>\alpha} \widetilde{\mathcal{G}}^{d}(f)^{p_{2}}\left(x^{\prime}, x_{m}\right) d x^{\prime} d x_{m}
\end{aligned}
$$

which, as above, shows that $\|T f\|_{H_{\mathrm{com}}^{p}} \leq C\|g(T f)\|_{p} \leq C\|f\|_{H_{\mathrm{com}}^{p}}$ for any $0<$ $p_{2}<p<p_{1}<\infty$.

\section{References}

[1] Chang, S. Y. A. and Fefferman, R.: Some recent developments in Fourier analysis and $H^{p}$-theory on product domains. Bull. Amer. Math. Soc. (N.S.) 12 (1985), no. 1, 1-43.

[2] Chang, S. Y. A. And Fefferman, R.: A continuous version of duality of $H^{1}$ with BMO on the bidisc. Ann. of Math. (2) 112 (1980), no. 1, 179-201.

[3] Chang, S. Y.A. And Fefferman, R.: The Calderón-Zygmund decomposition on product domains. Amer. J. Math. 104 (1982), no. 3, 455-468.

[4] Colfman, R. R. And Weiss, G.: Extensions of Hardy spaces and their use in analysis. Bull. Amer. Math. Soc. 83 (1977), no. 44, 569-645.

[5] Fefferman, C. And Stein, E. M.: $H^{p}$ spaces of several variables. Acta. Math. 129 (1972), no. 3-4, 137-193.

[6] Fefferman, R.: The atomic decomposition of $H^{1}$ in product spaces. Adv. Math. 55 (1985), 90-100.

[7] Fefferman, R.: Harmonic analysis on product spaces. Ann. of Math. (2) 126 (1987), no. 1, 109-130.

[8] Ferguson, S. And Lacey, M.: A characterization of product BMO by commutators. Acta Math. 189 (2002), no. 2, 143-160.

[9] Folland, G. B. And Stein, E. M.: Hardy spaces on homogeneous groups. Mathematical Notes 28, Princeton University Press, Princeton, N.J.; University of Tokyo Press, Tokyo, 1982.

[10] Frazier, M. and Jawerth, B.: A discrete transform and decomposition of distribution. J. Func.Anal. 93 (1990), no. 1, 34-170.

[11] Frazier, M., Jawerth, B. And Weiss, G.: Littlewood-Paley theory and the study of function spaces. CBMS Regional conference series in Mathematics 79, American Mathematical Society, Providence, RI, 1991.

[12] García Cuerva, J. And Rubio de Francia, J. L.: Weighted norm inequalities and related topics. North-Holland Math. Studies 116, North-Holland, Amsterdam, 1985. 
[13] Han, Y. And Lu, G.: Discrete Littlewood-Paley-Stein theory and multi-parameter Hardy spaces associated with flag singular integrals. Available at arXiv: 0801.1701 [math. CA].

[14] HAn, Y. ANd Lu, G.: Endpoint estimates for singular integral operators on multiparameter Hardy spaces associated with the Zygmund dilations. Preprint.

[15] Han, Y. and Lu, G.: Some recent works on multiparameter Hardy space theory and discrete Littlewood-Paley analysis. In Trends in partial differential equations, 99-191. Adv. Lect. Math. 10, Int. Press, Somerville, MA, 2010.

[16] Han, Y., Lu, G. And Sawyer, E.: Implicit multiparameter Hardy spaces and Marcinkiewicz multipliers on the Heisenberg group. Submitted.

[17] Journé, J.-L.: Calderón-Zygmund operators on product space. Rev. Mat. Iberoamericana 1 (1985), no. 3, 55-91.

[18] Pipher, J.: Journé's covering lemma and its extension to higher dimensions. Duke Math. J. 53 (1986), no. 3, 683-690.

[19] Phong, D. H. And Stein, E. M.: Some further classes of pseudodifferential and singular-integral operators arising in boundary valve problems. I. Composition of operators. Amer. J. Math. 104 (1982), 141-172.

[20] Wainger, S. And Weiss, G.: Hilbert transforms and maximal functions related to variable curves. Harmonic analysis in Euclidean spaces. In Proc. Sympos. Pure Math., Williams Coll., Williamstown, Mass., 1978, Part 1, 95-98. Proc. Sympos. Pure Math. 35, Amer. Math. Soc., Providence, RI, 1979.

Received July 12, 2011; revised February 9, 2012.

Yongshen Han: Department of Mathematics, Auburn University, Auburn, AL 36849, USA.

E-mail: hanyong@auburn.edu

Chincheng Lin: Department of Mathematics, National Central University, Taiwan, Chung-Li 320, Taiwan.

E-mail: clin@math.ncu.edu.tw

Guozhen Lu: Department of Mathematics, Wayne State University, Detroit, MI 48202, USA.

E-mail: gzlu@math. wayne.edu

Zhuoping Ruan: Department of Mathematics and IMS, Nanjing University, Nanjing, 210093, P. R. China.

E-mail: zhuopingruan@nju.edu.cn

ERIC T. SAWYeR: Department of Mathematics and Statistics, McMaster University, Hamilton, Ontario, L8S 4K1, Canada.

E-mail: sawyer@mcmaster.ca

The authors' research is supported by the NCU Center for Mathematics and Theoretic Physics, the NSC of Taiwan under Grant \#NSC 99-2115-M-008-002-MY3The, the NSF grant DMS0901761, the NSCF grant No. 11025105 and the Canadian NSERC grant, respectively. 